\title{
Probable member stars of the gravitational theory-testing globular clusters AM 1, Pal 3 and Pal 14 ${ }^{\star, \star \star}$
}

\author{
M. Hilker
}

Sternwarte der Universität Bonn, Auf dem Hügel 71, 53121 Bonn, Germany

e-mail: mhilker@astro.uni-bonn.de

Received 9 October 2005 / Accepted 17 October 2005

\section{ABSTRACT}

\begin{abstract}
Some of the Galactic outer halo globular clusters are excellent tools to probe gravitational theories in the regime of weak accelerations. The measurement of the line-of-sight velocity dispersion among stars in these clusters will differentiate between the validity of Newtonian dynamics (low velocity dispersion) and the possiblity of modified Newtonian dynamics (MOND) or dark matter dominated globular clusters (high velocity dispersion). In this paper, the properties of probable member stars of the three best-case gravitational theory-testing clusters AM 1, Pal 3 and $\mathrm{Pal} 14$ are presented. The member selection is based on VLT photometry in Johnson $B V$. The positions of the stars were determined with an accuracy of the order of $\leq 0$ '2, allowing their direct use for follow-up spectroscopy. The distance, reddening, age and metallicities of the clusters were estimated from isochrone fitting. Furthermore, improved structural parameters, like central coordinates, ellipticity, half-light radius, King model core and tidal radius, are presented.
\end{abstract}

Key words. Galaxy: globular clusters: individual: AM 1 - Galaxy: globular clusters: individual: Pal 3 Galaxy: globular clusters: individual: Pal 14 - astrometry - Galaxy: kinematics and dynamics

\section{Introduction}

In the distant halo of our Milky Way there exist several low mass, often diffuse globular clusters (GCs). Most of these outer halo GCs belong to the so-called "young halo clusters", comprising about 30 Milky Way GCs (e.g. Zinn 1993; Mackey \& Gilmore 2004). From their horizontal branch morphology it was estimated that they are about 1-2 Gyr younger than the "old halo clusters". Also they have on average larger half-light radii than the inner halo GCs (Mackey \& van den Bergh 2005). The similarity of their properties to those of external globular clusters (belonging to Milky Way satellite galaxies) led to the idea that they were accreted into the Galactic halo (e.g. van den Bergh \& Mackey 2004, originally proposed by Searle $\&$ Zinn 1978). The most remote halo GCs (at galacto-centric distances of $\geq 100 \mathrm{kpc}$ ) lie on eccentric orbits, mostly undisturbed by external tidal forces of our Galaxy.

Recently, it has been proposed by Baumgardt et al. (2005) that some of these distant Galactic globular clusters are excellent tools to probe gravitational theories in the regime of very weak accelerations. Their internal and external accelerations are significantly below the critical acceleration

\footnotetext{
* Based on observations obtained at the European Southern Observatory, Chile (Observing Programme 074.D-0187).

$\star \star$ Complete Appendix A is only available in electronic form at http://www. edpsciences.org
}

parameter $a_{0}$ of modified Newtonian dynamics (MOND, Milgrom 1983; Bekenstein \& Milgrom 1984). In case of MOND the internal velocity dispersion among the stars in these clusters would be significantly higher than in the Newtonian standard dynamics. A higher than Newtonian velocity dispersion could also be explained by the existence of dark matter in globular clusters (e.g. Mashchenko \& Sills 2005). The line-of-sight velocity dispersion of the 8 gravitational theorytesting GCs listed by Baumgardt et al. (2005) is expected to range between 0.5 and $1.1 \mathrm{~km}^{2} \mathrm{~s}^{-2}$ in the Newtonian case, whereas two times higher values ( 1.1 to $2.4 \mathrm{~km}^{2} \mathrm{~s}^{-2}$ ) are predicted for MOND. Such velocity differences can be measured with existing high resolution spectrographs, providing the observation of a statistical meaningful sample ( 20-30) of stars.

However, at the distance of the proposed GCs of between 28 and $122 \mathrm{kpc}$, high resolution spectroscopic observations of these feeble, low mass clusters are challenging. There exist only few evolved stars that are bright enough to be observed in reasonable integration times with 8-m class telescopes. Therefore, it is very useful to know the positions and photometric properties of these bright member stars beforehand.

We present those properties in this paper for three of the Baumgardt-sample GCs, namely AM 1, Pal 3 and Pal 14. These clusters were selected because they are expected to show the largest differences between Newtonian and MOND dynamics. They are therefore the best cases for testing gravitational 
Table 1. Observation log. The appendices "N" and "S" in the GC names denote the pointings North and South of their centers, respectively.

\begin{tabular}{|c|c|c|c|c|c|c|c|c|c|}
\hline \multirow[t]{2}{*}{ Name } & \multirow{2}{*}{$\begin{array}{c}\alpha(2000)^{a} \\
{[\mathrm{~h}: \mathrm{m}: \mathrm{s}]}\end{array}$} & \multirow{2}{*}{$\begin{array}{c}\delta(2000)^{a} \\
{\left[{ }^{\circ}:^{\prime}:^{\prime \prime}\right]}\end{array}$} & \multirow[t]{2}{*}{ Date } & \multicolumn{2}{|c|}{ Exposure times [s] } & \multicolumn{2}{|c|}{ Seeing ["] } & \multicolumn{2}{|c|}{ Airmass } \\
\hline & & & & $B$ & $V$ & $B$ & $V$ & $B$ & V \\
\hline AM 1-N & $03: 55: 02.6$ & $-49: 34: 41.9$ & 2005 Feb 15 & $3 \times 25$ & $3 \times 10$ & 0.6 & 0.7 & 1.20 & 1.20 \\
\hline AM 1-S & 03:55:02.6 & $-49: 38: 42.0$ & $2005 \mathrm{Feb} 16$ & $3 \times 30$ & $3 \times 15$ & 0.6 & 0.6 & 1.21 & 1.22 \\
\hline Pal 3-N & $10: 05: 31.4$ & $+00: 06: 25.9$ & 2005 Feb 15 & $3 \times 25$ & $3 \times 10$ & 0.6 & 0.7 & 1.24 & 1.23 \\
\hline Pal 3-S & $10: 05: 31.4$ & $+00: 02: 26.0$ & 2005 Feb 15 & $3 \times 25$ & $3 \times 10$ & 0.5 & 0.5 & 1.22 & 1.21 \\
\hline Pal 14-N & $16: 11: 05.0$ & $+14: 59: 38.0$ & 2005 Feb 15 & $3 \times 25$ & $3 \times 10$ & 0.6 & 0.7 & 1.46 & 1.45 \\
\hline Pal 14-S & $16: 11: 05.0$ & $+14: 55: 37.9$ & 2005 Feb 15 & $3 \times 25$ & $3 \times 10$ & 0.5 & 0.6 & 1.43 & 1.42 \\
\hline
\end{tabular}

${ }^{a}$ The coordinates indicate the pointing of the observations.

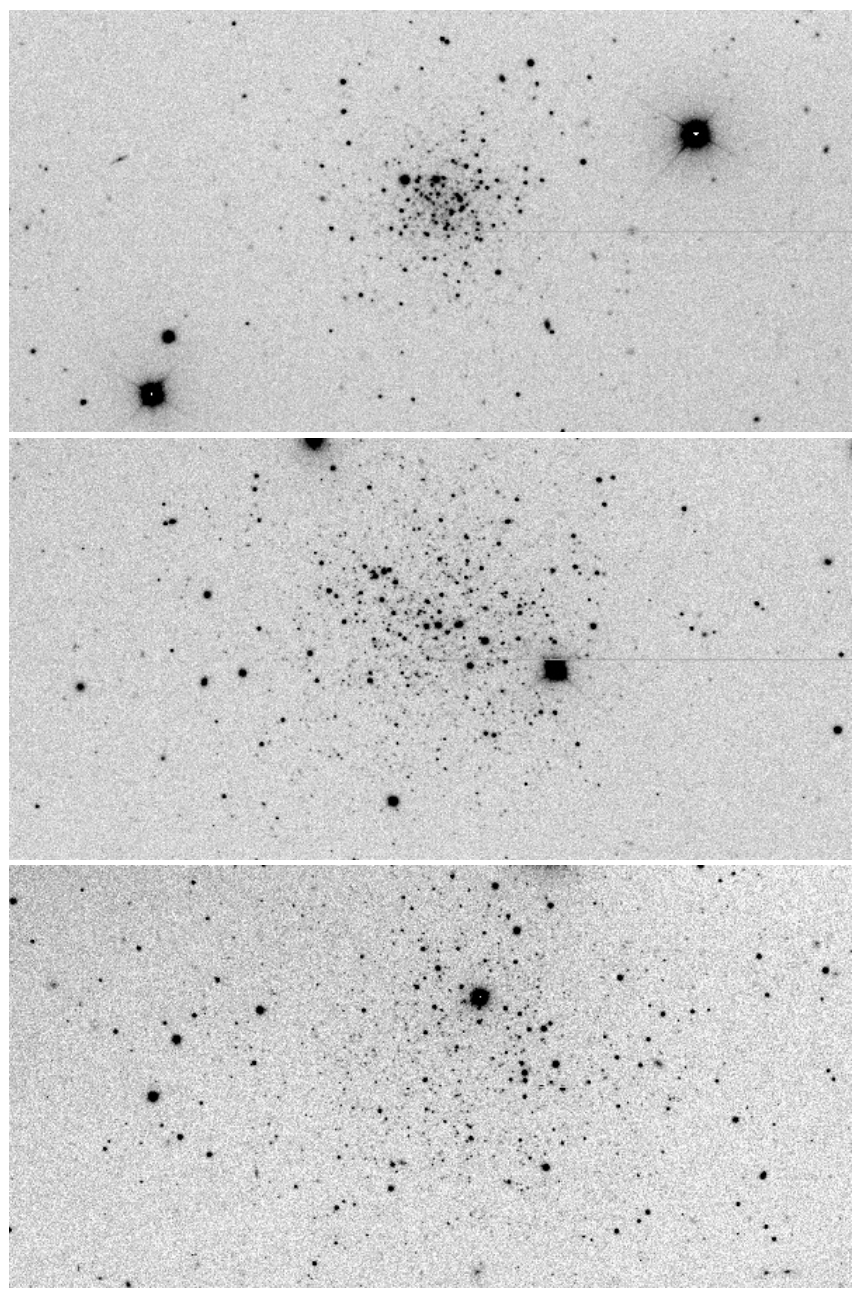

Fig. 1. Excerpts of the combined $V$ images for AM 1 (top), Pal 3 (middle) and Pal 14 (bottom). $4^{\prime} \times 2^{\prime}$ sections of the corresponding "master" chips are shown. North is up, East is left.

theories. Their basic parameters are given in Tables 3 and 4 (most of these parameters were taken from the 2003 version of Harris' list 1996). All three clusters belong to the "young halo" GCs with iron abundances between -1.5 and $-1.8 \mathrm{dex}$ and half-light radii in the range $17-25 \mathrm{pc}$. An age estimate for Pal 3 was derived from the detailed study of the colour magnitude diagram, especially the horizontal branch (HB) morphology. It seems that Pal 3 is between $1 \mathrm{Gyr}$
Table 2. Calibration coefficients for both nights. The zero points apply for magnitudes transformed from intensities in counts/sec. The signs are such that the following calibration equation applies: $\operatorname{mag}_{\mathrm{cal}}=$ $\operatorname{mag}_{\text {inst }}+Z P+k \times A M+C T \times(B-V)_{\text {cal }}$, with $\mathrm{ZP}=$ zero point, $\mathrm{k}=$ extinction coefficient, $\mathrm{AM}=$ airmass, and $\mathrm{CT}=$ colour term.

\begin{tabular}{lcrrr}
\hline \hline Chip & Filter & zero point & ext. coeff. & colour term \\
\hline chip1 & $V$ & $27.852 \pm 0.002$ & $-0.123 \pm 0.011$ & $0.034 \pm 0.008$ \\
& $B$ & $27.295 \pm 0.009$ & $-0.185 \pm 0.016$ & $-0.031 \pm 0.006$ \\
chip2 & $V$ & $27.841 \pm 0.012$ & $-0.162 \pm 0.018$ & $0.020 \pm 0.004$ \\
& $B$ & $27.298 \pm 0.008$ & $-0.208 \pm 0.016$ & $-0.033 \pm 0.006$ \\
\hline
\end{tabular}

(van den Berg 2000; Catelan et al. 2001) and 2 Gyr (Stetson et al. 1999) younger than ordinary inner halo GCs like M3. Also for Pal 14 a 3 to $4 \mathrm{Gyr}$ younger age than for typical GCs was estimated from the position of the main sequence turnoff (MSTO) relative to the HB level (Sarajedini 1997).

In this paper, we provide accurate positions, $V$ magnitudes and $(B-V)$ colours of the evolved stars in the three clusters AM 1, Pal 3 and Pal 14. This photometry is necessary and very useful for spectroscopic follow-up observations of these best-case gravitational theory-testing clusters.

\section{Observations and data reduction}

The observations were performed in February 2005 with the VLT/UT1 at Paranal (ESO), Chile. The instrument in use was the FORS 2 camera with a $4 \times 4 \mathrm{k}$ MIT CCD array attached. The data were read out with a $2 \times 2$ binning, resulting in a spatial scale of $0.25^{\prime \prime} /$ pixel and a field of view of $6.8 \times 6.8$.

Each of the three globular clusters AM 1, Pal 3 and Pal 14 was observed through the Johnson $B$ and $V$ filters under mostly photometric and very good seeing conditions at two slightly different positions. Once, centering the cluster on the "master" $4 \times 2 \mathrm{k}$ chip (also called "chip1"), and once on the "slave" $4 \times 2 \mathrm{k}$ chip (="chip2") of the $1 \times 2 \mathrm{CCD}$ array. Excerpts of the $V$ exposures on the "master" chip are shown in Fig. 1. The exposure times were chosen such that the brightest cluster red giants were not saturated. Three dithered integrations per filter and field were taken. For the observation log see Table 1.

The CCD frames were processed with standard IRAF routines. After bias subtraction and flatfield correction, the pixel shifts of the dithered images were determined. Then the shift 


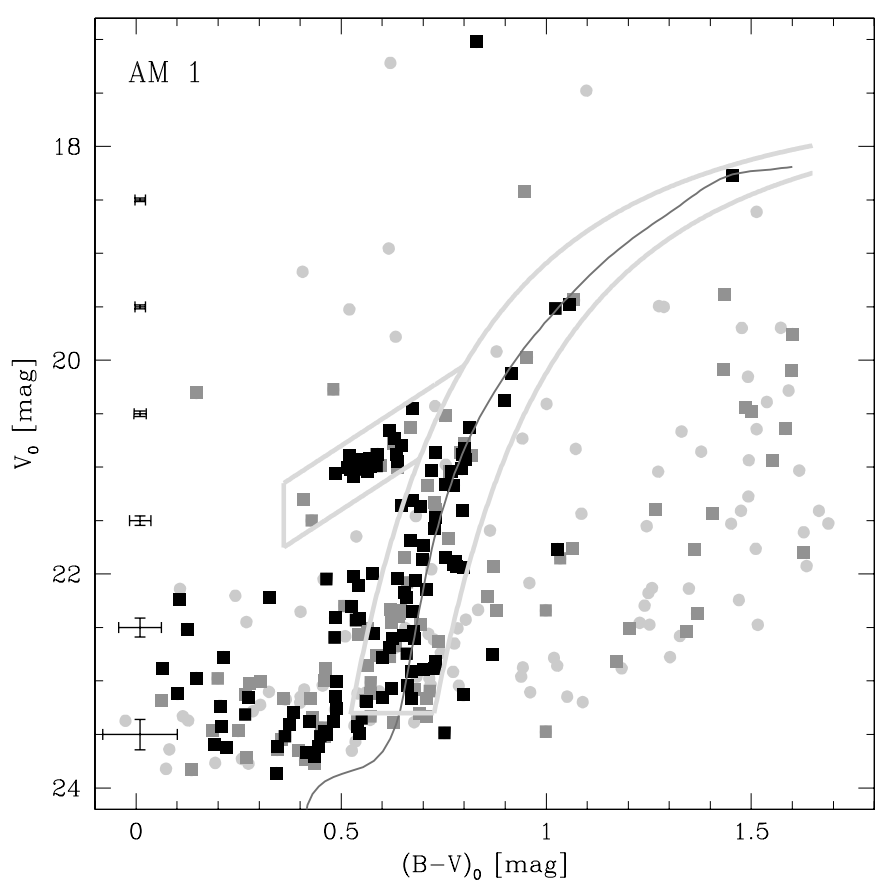

Fig. 2. Colour magnitude diagram of stars around AM 1. The reddening is negligible: $E_{B-V}=0.0$ mag. Dark grey squares are those objects that are located within five half-light radii from the centre of the cluster, black squares those within one half-light radius (see Fig. 3). Typical photometric errors are shown on the left. The selection areas for probable member stars are marked (red giant branch and horizontal branch). Overplotted is a Yonsei-Yale isochrone (Kim et al. 2002) with the following parameters: age $=11 \mathrm{Gyr},[\mathrm{Fe} / \mathrm{H}]=-1.4 \mathrm{dex},[\alpha / \mathrm{Fe}]=$ $0.3 \mathrm{dex}$. The isochrone has been shifted with $(m-M)_{V, 0}=20.45 \mathrm{mag}$ and $(B-V)_{\text {corr. }}=-0.01 \mathrm{mag}$ (see text for further details).

corrected frames were averaged using a clipping algorithm to exclude cosmics.

\section{Photometry and astrometry}

\subsection{Photometry}

The instrumental magnitudes of the stars were derived from PSF (point spread function) photometry using DAOPHOT II (Stetson 1987, 1992). For the comparison with the standard stars, aperture-PSF shifts were determined in all fields and filters. The amplitude of this shift ranges between 0.20 and $0.44 \mathrm{mag}$, its uncertainty is of the order of $0.02 \mathrm{mag}$.

In the first night, Landolt standard stars were observed. The coefficients of the calibration equation were taken from the ESO quality control program (see http://www . eso.org/ observing/dfo/quality/index_fors2.html). An overview of the calibration coefficients is given in Table 2.

The calibrated magnitudes of stars in the overlapping regions of the North and South fields were compared with each other. Shifts of the order of $\pm 0.02 \mathrm{mag}$ were found between the data sets. This is consistent with the uncertainty in the aperturePSF shift, but could also have been caused by the not totally photometric conditions during the observing run. Thus, the data set that was observed under better photometric conditions has

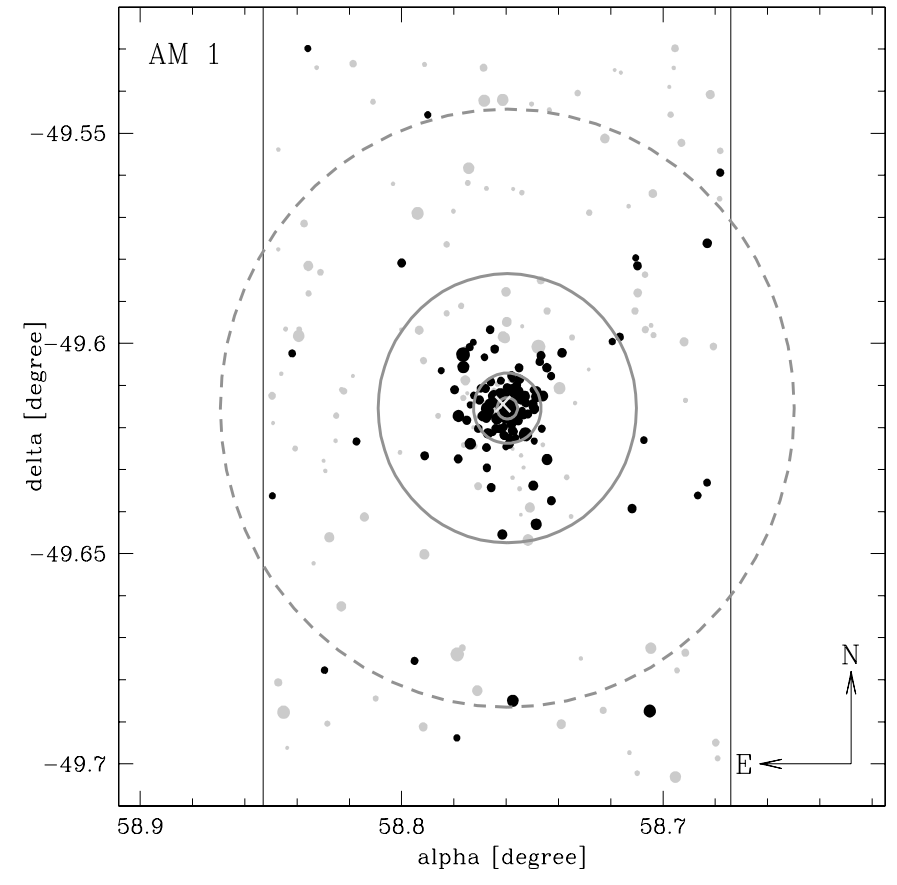

Fig. 3. Coordinates of stars around AM 1. The symbol size corresponds to the luminosity of an objects: the larger the dot the brighter the star. Black dots are probable member stars according to their position in the CMD (see highlighted areas in Fig. 2). The solid circles are from inside out the core radius, the half-light radius and the tidal radius (Harris 1996). The dashed circle is the tidal radius as calculated by Baumgardt et al. (2005). All circles are centred on the newly determined central coordinates of AM 1 (see Table 3). The cross marks the central coordinate from the list of Harris (Harris 1996). The vertical lines indicate the borders of our field-of-view.

been taken as the reference system for each cluster, and the magnitudes of the other set were shifted to this system. The magnitudes of stars that were measured in both data sets then were averaged for the final catalog.

After the photometric reduction, calibration of the magnitudes and combining of the data sets, the average photometric errors for red giants at $V=23$ were of the order of 0.08 in $V$ and 0.12 mag in $B$.

\subsection{Astrometry}

The positions of the cluster stars in terms of right ascension and declination (epoch J2000) were determined by deriving an astrometric solution between pixel and RA/Dec coordinates for stars of the US Naval Observatory (USNO) in the field-of-view. Each CCD chip was considered separately. Typically, between 60 and 80 USNO catalog stars were identified in chip1, and 40-60 in chip2. The IRAF task GEOMAP was used to find the astrometric solution. A "general" fit with full cross-terms of second order gave the best results. The rms of the fit in right ascension is 0 !' 17 for AM 1 and Pal 14, and 0!'24 for Pal 3. The accuracy in declination is $0{ }^{\prime} 14,0{ }^{\prime} 17$, and $0 !^{\prime} 19$ for AM 1, Pal 14, and Pal 3, respectively. 


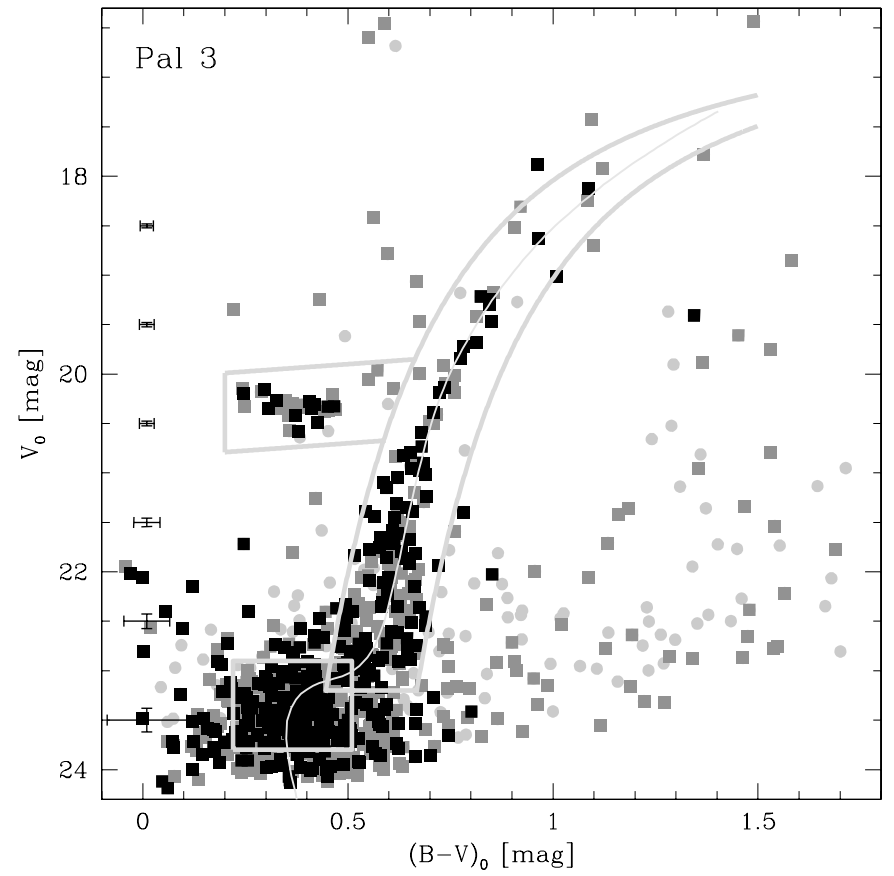

Fig. 4. Reddening corrected CMD of stars around $\mathrm{Pal} 3\left(E_{B-V}=0.040\right.$ and $A_{\mathrm{V}}=0.124 \mathrm{mag}$ ). Symbols and areas as in Fig. 2. Additionally, the area of main sequence turnoff stars is marked. The isochrone parameters are: age $=10 \mathrm{Gyr},[\mathrm{Fe} / \mathrm{H}]=-1.7 \mathrm{dex},[\alpha / \mathrm{Fe}]=0.3 \mathrm{dex}$, $(m-M)_{V, 0}=19.95 \mathrm{mag}$, and $(B-V)_{\text {corr. }}=-0.02 \mathrm{mag}$.

The coordinates of the member stars are listed in the Tables A.1-A.3. The derived coordinates also were used for the graphs of Figs. 3, 5, and 7.

\section{Distance, reddening, age and metallicity}

The calibrated and de-reddened magnitudes are presented in the colour-magnitude diagrams (CMDs, see Figs. 2, 4, and 6). The foreground reddening has been corrected with the $E_{B-V}$ values given in the Harris list (Harris 1996), and $A_{\mathrm{V}}=$ $3.1 E_{B-V}$. Only stars with photometric errors less than $0.15 \mathrm{mag}$ in $V$ and $B$ and ALLSTAR sharp and chi values in the ranges $-0.7<$ sharp $<1.0$ and chi $<3.0$ are shown. The red giant branch (RGB) and horizontal branch (HB) are clearly visible in all clusters. For Pal 3, even the main sequence turnoff (MSTO) region is well definded.

The distances to the clusters are derived from the apparent magnitude of the horizontal branch. We selected only those HB stars located within five half-light radii from the clusters' centres and which lie on the horizontal part of the HB at $(B-V)_{0} \sim 0.5$ (to avoid AGB and extended HB stars). The average HB magnitudes in $V$ and the corresponding distance moduli are given in Table 3 . They agree within \pm 0.03 mag with the values listed by Harris (1996).

In order to estimate the age and metallicity of the clusters we fitted the location and shape of the RGBs by appropriate isochrones. The Yonsei-Yale isochrone set by Kim et al. (2002) was used. An alpha-abundance of $[\alpha / \mathrm{Fe}]=0.3$ dex was adopted. The best-fitting isochrones are shown in Figs. 2, 4

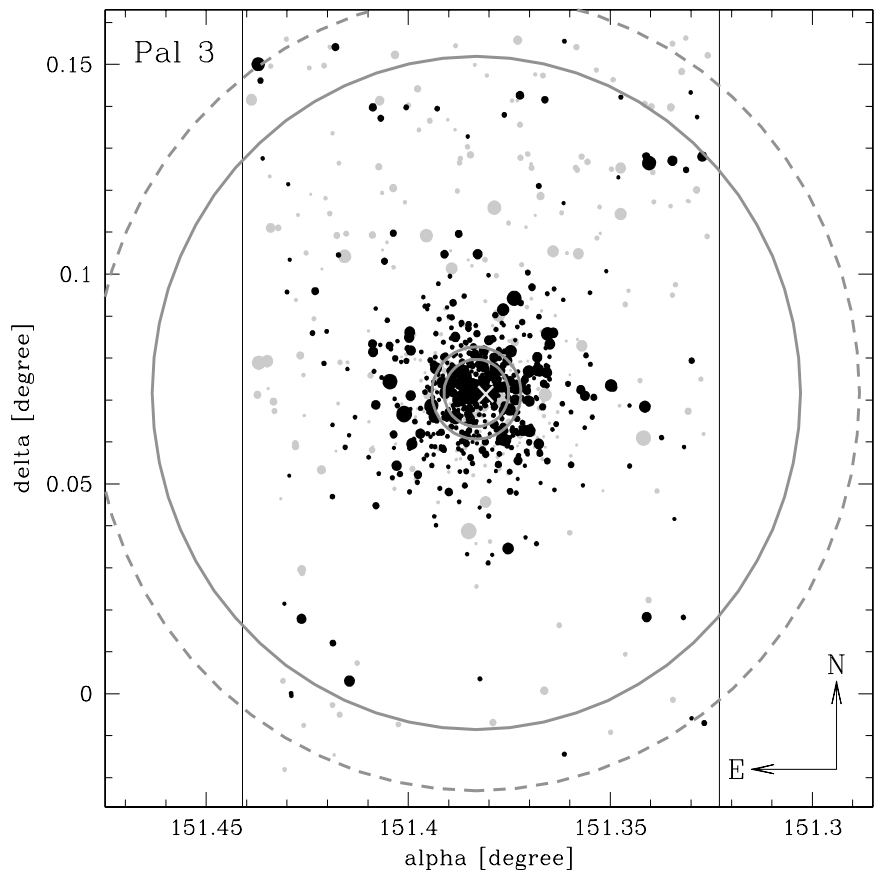

Fig. 5. Coordinates of stars around Pal3. For the explication of symbols, see Fig. 3.

and 6. They have ages between 10 and $11 \mathrm{Gyr}$, and metallicities in the range -1.7 to -1.4 dex. For Pal 3 and Pal 14 the best-fitting metallicity value agrees well with the spectroscopic values in the Harris list (see Table 3). For AM 1, no good isochrone fit with a metallicity of -1.8 dex could be found. Instead, a metallicity of -1.4 dex represents the shape of the RGB well. Either the spectroscopic abundance is not accurate enough and this cluster indeed has a higher metallicity or the isochrone fit is not constrained well enough. Besides the selection of the best-fitting ages and metallicities, the isochrones had to be slightly shifted in $V$ as well as in $(B-V)$ to match the exact location of the RGB. These corrections are given in the captions of the CMD figures, $(m-M)_{V, 0}$ and $(B-V)_{\text {corr. }}$, and in Table 3 (with $E_{B-V \text {,this work }}=E_{B-V \text {,Harris }}+(B-V)_{\text {corr. }}$ ). These corrections are of the order of the photometric errors and within the uncertainties of not perfectly photometric conditions.

\subsection{Member selection}

The main purpose of this paper is to provide a list of bright cluster members for follow-up spectroscopy. Therefore, probable cluster members have to be selected. The principle criterium is that member stars should be located close to the isochrone in the colour-magnitude diagram. A second criterium is their distance to the cluster centre. In the Figs. 2, 4 and 6 the regions of probable member stars are highlighted. The RGB and HB regions are marked for all clusters. In Pal 14, an additional region of subgiant branch (SGB) stars has been selected, in Pal 3 also the MSTO region. Furthermore, stars within one and five half-light radii are shown in the graphs as black and dark grey squares, respectively. The core radius, half-light 


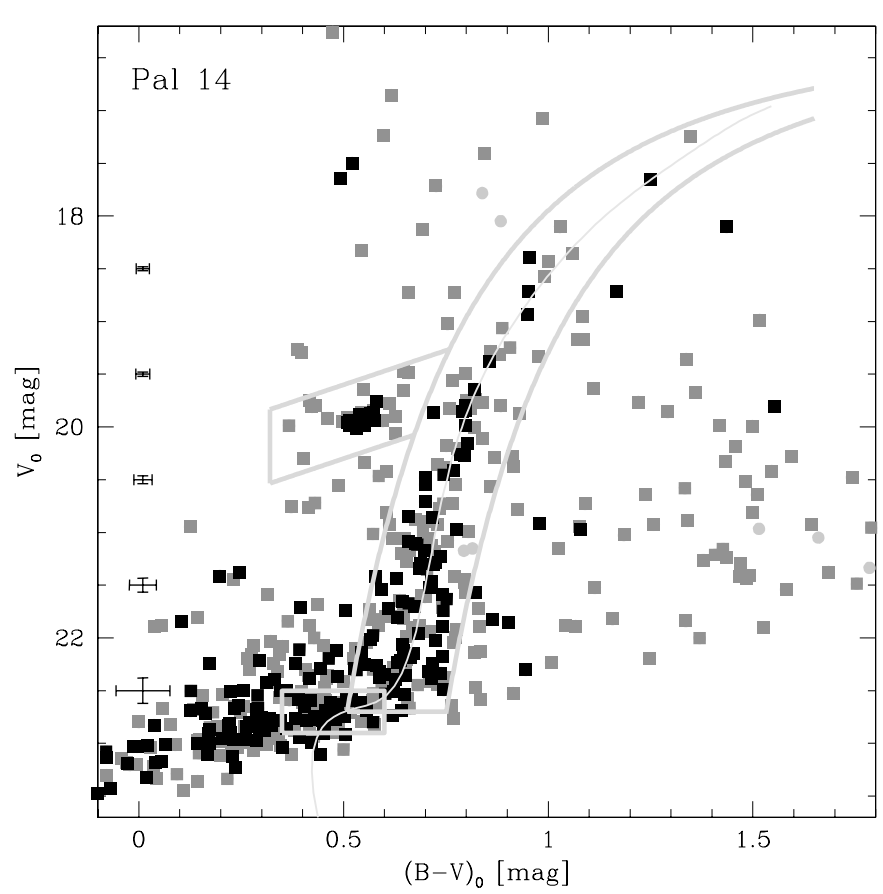

Fig. 6. Reddening corrected CMD of stars around Pal $14\left(E_{B-V}=\right.$ 0.040 and $\left.A_{\mathrm{V}}=0.124 \mathrm{mag}\right)$. Symbols and areas as in Fig. 2. The lowest area includes probable subgiant branch stars. The isochrone parameters are: age $=10 \mathrm{Gyr},[\mathrm{Fe} / \mathrm{H}]=-1.5 \mathrm{dex},[\alpha / \mathrm{Fe}]=0.3 \mathrm{dex}$, $(m-M)_{V, 0}=19.42 \mathrm{mag}$, and $(B-V)_{\text {corr. }}=0.03 \mathrm{mag}$.

radius and tidal radius (Harris 1996; Baumgardt et al. 2005) are listed in Table 4 and shown in Figs. 3, 5 and 7.

In the Appendix, magnitude limited samples of probable member stars are listed (Tables A.1-A.3). Only stars within the tidal radius given by Baumgardt et al. (2005) have been considered. The magnitude limits are $V_{0}=23.0,22.5$, and 22.2 for AM 1, Pal 3, and Pal 14, respectively. Thus, only RGB, HB and AGB stars are contained in the lists. Note: the full tables only are availble in the online version of the article.

\section{Structural parameters}

Although the photometry of the observed clusters is not very deep and we do not sample the main sequence stars, some structural parameters of the globular clusters can be derived from the selected evolved member stars.

\subsection{Cluster centres}

The cluster centres were determined by analysing the number distribution of probable member stars as function of right ascension and declination. Probable member stars are those of the highlighted regions in the CMDs (see Figs. 2, 4, and 6). Furthermore, the member stars have been restricted to an approximately square region $\left(6.5^{\prime} \times 6.5^{\prime}\right)$ around their apparent cluster centres. The number distributions of the stars are shown in Fig. 8 as histograms and as binning independent representations (using an Epanechnikov kernel). The binning independent number distributions were fitted by Gaussians. The

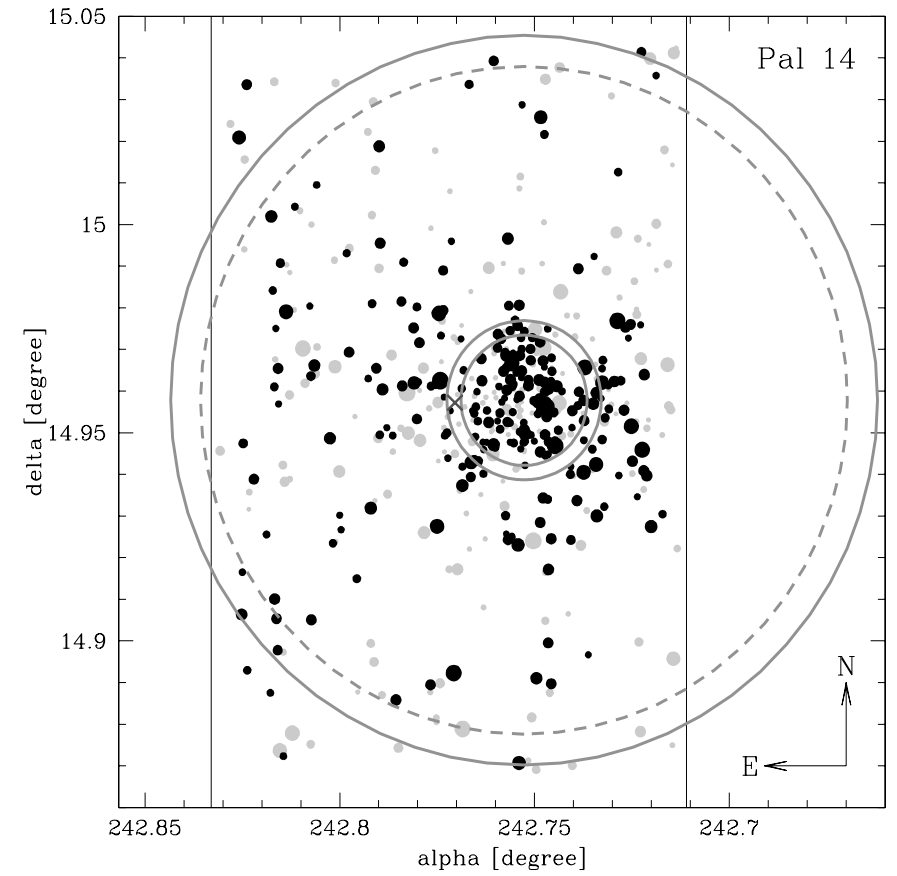

Fig. 7. Coordinates of stars around Pal 14. For the explication of symbols, see Fig. 3.

peak of these Gaussians defines the cluster centre. In Table 3 the derived values are listed. The accuracy of the centre determination is about \pm 2 ". While the declination of the centres agree very well with those listed in the Harris list, there are some notable deviations in the right ascension (see also crosses in Figs. 3, 5, and 7). Especially, the right ascension listed for Pal 14 is off by $\sim 62^{\prime \prime}$ with respect to our value. We are confident that our values are very accurate and suggest correcting the coordinates in the "official" lists (e.g. Harris 1996).

\subsection{Angular distribution and ellipticity}

The ellipticity of the clusters is of interest because rotation or the tidal loss of stars in opposite directions might cause a detectable signal. To investigate this we studied the angular distribution of probable member stars including the main sequence turnoff region stars (see Fig. 9). We choose the radial range to be from outside the core radius (only for AM 1 and Pal 3) to avoid crowding effects to inside the maximum radius of the observed CCD area to guarantee a homogeneous angular coverage. The angular distributions (counted in bins of 20 degree) then were fitted by a double-cosine function of the form $N=A+B \cdot \cos (2 \cdot(\phi+C))$, where $A$ and $B$ define the ellipticity as follows: $\epsilon=(A-B) /(A+B)$, assuming that the ellipticity is expressed as $1-b / a$ (a: semi major axis, $b$ : semi minor axis). The error for the ellipticity was propagated from the Poisson statistics of the number counts and the rms of the fit. The results are: $\epsilon_{\mathrm{AM} 1}=0.20 \pm 0.26, \epsilon_{\mathrm{Pal} 3}=0.11 \pm 0.20$, and $\epsilon_{\mathrm{Pal} 14}=0.13 \pm$ 0.10 . None of the clusters exhibits a significant ellipticity. The distribution of their stars is compatible with a homogeneous, circular distribution within the errors. 
Table 3. Basic photometric parameters of AM 1, Pal 3, and Pal 14. The second row for each cluster gives the values from Harris' list (1996). $E_{B-V, S 98}$ is the reddening value by Schlegel et al. (1998). The magnitude of the horizontal branch $V_{\mathrm{HB}}$ and the total apparent $V$ magnitude $V_{\text {tot }}$ are not corrected for extinction. The first distance modulus is based on the derivation of the absolute magnitude of the HB using $M_{V, \mathrm{HB}}=$ $0.15[\mathrm{Fe} / \mathrm{H}]+0.8$, the second one on the isochrone fitting. The distance to the Sun $R_{\text {Sun }}$ was calculated from the first distance modulus after correction for extinction with $A_{V}=3.1 E_{B-V, S 98}$.

\begin{tabular}{cccccccccccc}
\hline \hline Name & $\begin{array}{c}\mathrm{RA}(2000) \\
{[\mathrm{h}: \mathrm{m}: \mathrm{s}]}\end{array}$ & $\begin{array}{c}\operatorname{Dec}(2000) \\
{\left[{ }^{\circ}:^{\prime}:^{\prime \prime}\right]}\end{array}$ & $\begin{array}{c}E_{B-V, \mathrm{~S} 98} \\
{[\mathrm{mag}]}\end{array}$ & $\begin{array}{c}E_{B-V} \\
{[\mathrm{mag}]}\end{array}$ & $\begin{array}{c}V_{\mathrm{HB}} \\
{[\mathrm{mag}]}\end{array}$ & $\begin{array}{c}(m-M)_{V, \mathrm{HB}} \\
{[\mathrm{mag}]}\end{array}$ & $\begin{array}{c}(m-M)_{V, \text { iso }}[\mathrm{Fe} / \mathrm{H}] \\
{[\mathrm{mag}]}\end{array}$ & $\begin{array}{c}R_{\text {Sun }} \\
{[\mathrm{dex}]}\end{array}$ & $\begin{array}{c}V_{\text {tot }}{ }^{a} \\
{[\mathrm{kpc}]}\end{array}$ & $\begin{array}{c}M_{V} \\
{[\mathrm{mag}]}\end{array}$ & {$[\mathrm{mag}]$} \\
\hline \multirow{2}{*}{ AM1 } & $03: 55: 02.3$ & $-49: 36: 55$ & 0.01 & $\ldots$ & 20.98 & 20.39 & 20.45 & -1.40 & 118.0 & 15.84 & -4.55 \\
& $03: 55: 02.7$ & $-49: 36: 52$ & $\ldots$ & 0.00 & 20.96 & 20.43 & $\ldots$ & -1.80 & 121.9 & 15.72 & -4.71 \\
\multirow{2}{*}{ Pal3 } & $10: 05: 31.9$ & $00: 04: 18$ & 0.04 & 0.02 & 20.48 & 19.94 & 19.95 & -1.70 & 91.9 & 14.91 & -5.03 \\
& $10: 05: 31.4$ & $00: 04: 17$ & $\ldots$ & 0.04 & 20.51 & 19.96 & $\ldots$ & -1.66 & 92.7 & 14.26 & -5.70 \\
Pal 14 & $16: 11: 00.6$ & $14: 57: 28$ & 0.03 & 0.07 & 20.03 & 19.46 & 19.42 & -1.50 & 74.7 & 14.68 & -4.78 \\
& $16: 11: 04.9$ & $14: 57: 29$ & $\ldots$ & 0.04 & 20.04 & 19.47 & $\ldots$ & -1.52 & 73.9 & 14.74 & -4.73 \\
\hline
\end{tabular}

${ }^{a}$ The total magnitude in the first row for each cluster is based on the integrated luminosity of probable member stars down to the magnitude limits of the individual data sets. It therefore excludes most of the bright foreground stars, but also the fainter main sequence stars.

Table 4. Basic structural parameters of AM 1, Pal 3, and Pal 14. The second row for each cluster gives the values from Harris' list (1996). The last three columns are physical scales based on the distances in Table 3.

\begin{tabular}{ccccccccc}
\hline \hline Name & $\begin{array}{c}\mu_{V, \text { central }} \\
{\left[\mathrm{mag} / \mathrm{arcsec}^{2}\right]}\end{array}$ & $\begin{array}{c}r_{\mathrm{c}}{ }^{a} \\
{\left[^{\prime}\right]}\end{array}$ & $\begin{array}{c}r_{\mathrm{h}} \\
{\left[^{\prime}\right]}\end{array}$ & $\begin{array}{c}r_{\mathrm{t}}{ }^{a} \\
{\left[^{\prime}\right]}\end{array}$ & $\mathrm{c}^{a, b}$ & $\begin{array}{c}r_{\mathrm{c}} \\
{[\mathrm{pc}]}\end{array}$ & $\begin{array}{c}r_{\mathrm{h}} \\
{[\mathrm{pc}]}\end{array}$ & $\begin{array}{c}r_{\mathrm{t}} \\
{[\mathrm{pc}]}\end{array}$ \\
\hline AM1 & 23.11 & $0.18(0.14)$ & 0.32 & $3.24(3.29)$ & $1.26(1.37)$ & $6.2(4.8)$ & 11.0 & $111.3(113.0)$ \\
& 23.86 & 0.15 & 0.50 & $1.92(4.27)$ & 1.12 & 5.3 & 17.7 & $68.1(151.6)$ \\
Pal 3 & 23.84 & $0.54(0.40)$ & 0.72 & $3.34(3.72)$ & $0.79(0.97)$ & $14.4(10.7)$ & 19.3 & $89.3(99.5)$ \\
& 23.08 & 0.48 & 0.66 & $4.81(5.69)$ & 1.00 & 12.9 & 17.8 & $129.8(153.5)$ \\
Pal 14 & 24.92 & $0.70(1.14)$ & 1.28 & {$[5.00]^{c}$} & $0.85(0.59)$ & $15.2(24.8)$ & 27.8 & {$[108.7]^{c}$} \\
& 25.55 & 0.94 & 1.15 & $5.26(4.81)$ & 0.75 & 20.21 & 24.7 & $113.1(103.4)$ \\
\hline
\end{tabular}

${ }^{a}$ In brackets the values of the fit to the surface brightness profile are given (first row for each cluster). The tidal radii in brackets of the second row for each cluster are the values from Baumgardt et al. (2005).

${ }^{b}$ Concentration parameter: $c=\log \left(r_{\mathrm{t}} / r_{\mathrm{c}}\right)$.

${ }^{c}$ This value was not derived but adopted.

\subsection{Surface density profiles}

The probable member stars of the three clusters are evolved stars of more or less the same mass. Since they are the brightest stars of the clusters they dominate the surface brightness of those clusters. One can derive the structural parameters of the clusters by fitting density profiles to either the number density distribution or the surface brightness distribution of the stars. This is shown in Fig. 10. The number density counts have been corrected for background/foreground field star contamination by estimating the field star number counts from 0.5 arcmin wide strips in the northern and southern edge of the observed CCD areas. The number density of contaminating field stars in the selected CMD areas is $0.15,0.77$, and 0.62 objects/ $\mathrm{arcmin}^{-2}$ for AM 1, Pal 3, and Pal 14, respectively. The core radius, the tidal radius, and the central surface brightness of the clusters were determined from the best fitting King (King 1962) profiles. For Pal 14, the tidal radius had to be fixed to 5 arcmin (the mean value between Harris 1996 and Baumgardt et al. 2005) since it could not be constrained by the fit. The half-light radii were derived from a curve-of-growth analysis of the integrated light of the member stars. The results of our analyses are given in Table 4. Typical errors are of the order 0’06 (AM 1, Pal 3) and 0'.20 (Pal 14) for $r_{\mathrm{c}}, 0 \mathbf{\prime}^{\prime}$.04 (Pal 3) and 0 '.19 (AM 1) for $r_{\mathrm{t}}$, while for $r_{\mathrm{h}}$ they are $\sim 0$.'02 respectively. The errors for the central surface brightness are between 0.25 and $0.40 \mathrm{mag}$.

Not all our derived values agree with the ones listed by Harris (1996) and/or Baumgardt et al. (2005). For AM 1, we find a slightly smaller half-light radius, but a significantly larger tidal radius than listed by Harris. A small tidal radius does not fit the profile. Also the the central surface brightness is much higher than Harris' value. In the contrary, for Pal 3, we derive a slightly larger half-light radius, but a smaller tidal radius. Also for Pal 14, a larger half-light radius fits the profile best.

Note however that all our values are valid for the selected probable member stars. Adding the fainter, lower mass main sequence stars would raise the central surface brightness slightly. One might think that including the faint stars would increase the characteristic radii since these stars might be more widely distributed than the more massive, evolved stars due to the 

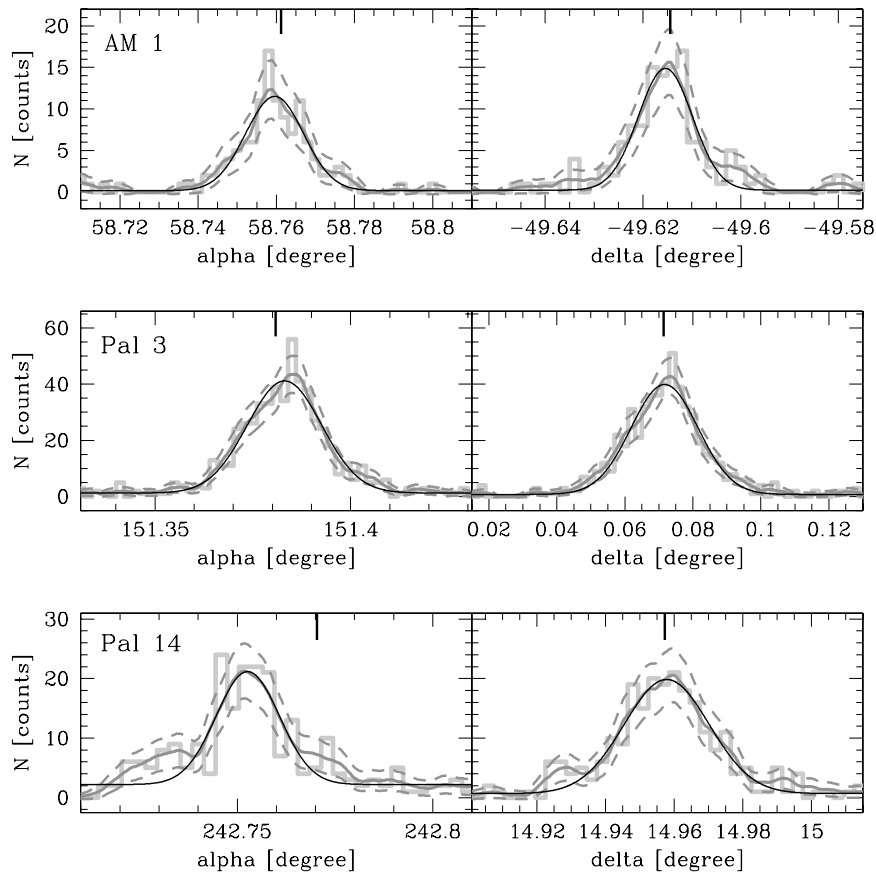

Fig. 8. Determination of globular cluster centres from the spatial distribution of selected probable member stars (see the CMDs). The grey histograms in the left panels show the distribution in West-East-direction, the ones in the right panels the distribution in South-North-direction. Binning independent representations of the counts (Epanechnikov kernel of 0.002 (AM 1 and Pal3) and 0.003 (Pal 14) degree width) are overplotted as thin curves, together with their $1 \sigma$ uncertainty limits (dashed curves). The thicklined Gaussians are fits to the binning independent distributions. The bold tickmarks on top of each plot mark the central positions from the Harris catalog (1996).

dynamical evolution of the cluster. However, the half-mass relaxation times of the clusters are between 5.5 and $9.1 \mathrm{Gyr}$, close to a Hubble time. This suggests that dynamical evolution does not play a major role in the shaping of the clusters.

\section{Conclusions}

The outer halo globular clusters AM 1, Pal 3, and Pal 14 were imaged with FORS2 on the VLT under good seeing and mostly photometric conditions. Accurate PSF photometry in Johnson $B V$ of stars brighter than $V=24$ mag was performed in $6.8 \times 10$ '.8 large fields around these clusters. The absolute position of the stars was determined with an accuracy of $<0.2^{\prime \prime}$ by applying a general astrometric solution derived from stars of the USNO catalog in the observed fields. This positional accuracy is sufficient to be used in input catalogs for most of the available multi-object spectrographs (using either fibres or slit masks).

Probable member stars of the clusters were selected according to their position in the colour-magnitude diagram and their distance to the cluster centres. The positions and photometric properties ( $V$ magnitude, $(B-V)$ colour) of all probable member stars within the tidal radius (as calculated by

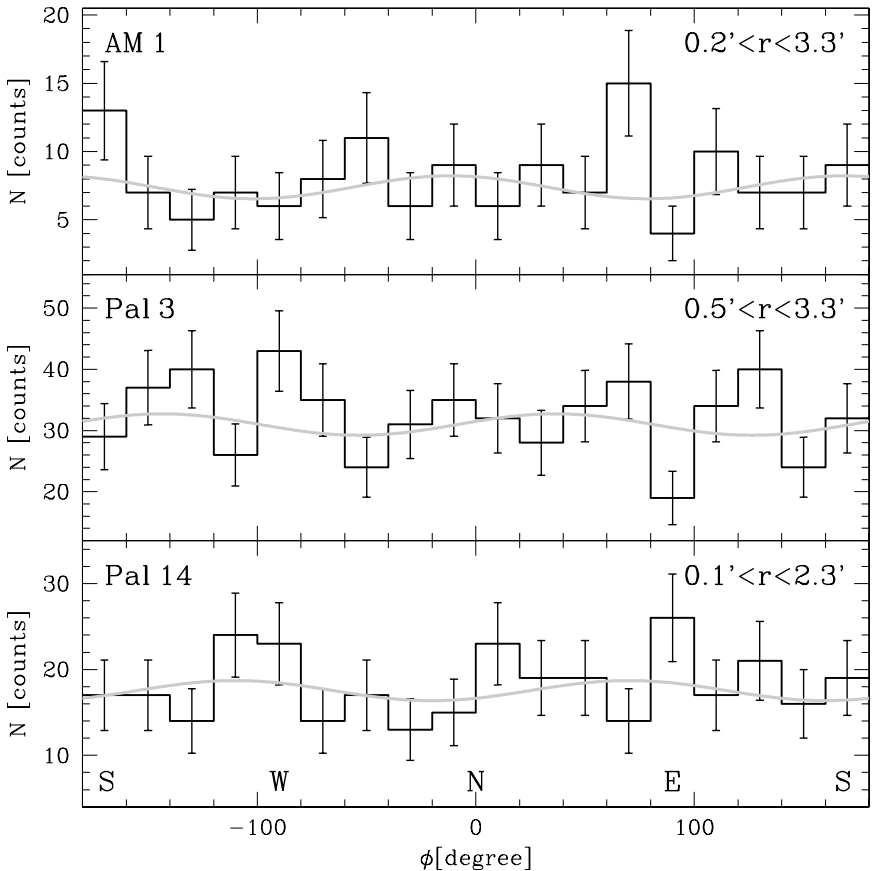

Fig. 9. The angular distribution of probable member stars in the indicated radial ranges around the three clusters together with statistical errorbars. The grey curves are best-fitting double-cosines, indicating the ellipticity of the clusters.
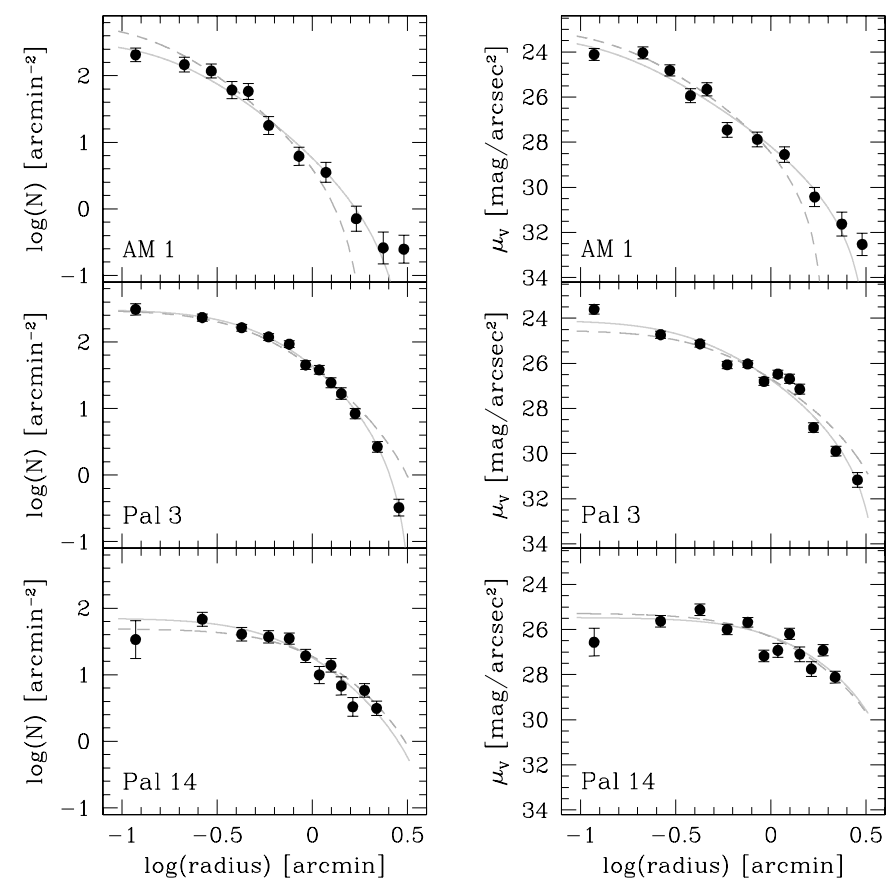

Fig. 10. The surface number density distribution (left panels) and the surface brightness distribution (right panels) of probable member stars is shown for the three clusters. The grey curves are best-fitting King models. Dashed curves are fits to the distributions when adopting the values for $r_{\mathrm{c}}$ and $r_{\mathrm{t}}$ of Harris' list (1996). The parameters of both fits are given in Table 4.

Baumgardt et al. 2005) and brighter than $V_{0}=23.0,22.5$, 22.2 mag for AM 1, Pal3, and Pal 14, respectively, are presented in the tables of the appendix. Although challenging, 
one can reach with modern high resolution spectrographs, like UVES at the VLT, a signal-to-noise above 10 for stars as faint as $V=20.5 \mathrm{mag}$ within one observing night. This limit would mean a sample size of 9,48 , and 72 probable member stars for AM 1, Pal3, and Pal 14, respectively. Except for AM 1, these sample sizes are sufficient to derive an accurate radial velocity dispersion of the clusters.

The colour-magnitude diagrams have been used to estimate - by isochrone fitting - distances, reddenings, ages and metallicities to the three clusters. Our derived values for the horizontal branch magnitude $V_{\mathrm{HB}}$, reddening and distance modulus $(m-M)_{V}$ generally agree within \pm 0.03 mag with the values given in the literature (AM 1: Madore \& Freedman 1989; Pal 3: Stetson et al. 1999; Pal 14: Holland \& Harris 1992). Only for Pal 14 do we get a slightly better isochrone fit when adopting a $0.05 \mathrm{mag}$ shorter distance modulus and a 0.03 mag higher reddening (see Table 3 ). Since the turnoff regions are not very well constrained in the CMDs of the three clusters, age estimates have to be taken with caution. However, we found that isochrones with ages of 10-11 Gyr fit the turnoff-SGB-RGB transition region better than those of older ages. Our age estimates are consistent with the idea that the three clusters belong to the "young halo" GCs which are, on average, 1-2 Gyr younger than the old "inner halo" GCs (e.g. Mackey \& Gilmore 2004). The metallicity of the chosen isochrones is mainly constrained by the shape of the red giant branch. The adopted values for Pal $3(-1.7$ dex $)$ and Pal $14(-1.5 \mathrm{dex})$ agree well with those given in the literature: Pal 3: -1.78 (Zinn 1985), $-1.57 \pm 0.3$ (Armandroff et al. 1992), $-1.70 \pm 0.15$ (Ortolani \& Gratton 1989); Pal 14: $-1.47 \pm$ 0.3 (Zinn 1985), $-1.60 \pm 0.18$ (Armandroff et al. 1992). The isochrone of AM 1, however, fits the RGB better with a higher metallicity $(-1.4$ dex $)$ than derived in previous work: -1.69 (Zinn 1985), $-1.7 \pm 0.2$ (Suntzeff et al. 1985). New spectroscopic metallicities are needed to decide whether AM 1 really is more metal-rich than thought before.

From the spatial distribution of probable member stars in AM 1, Pal3, and Pal 14 structural parameters of the clusters have been determined and compared to the values given in Harris' list (1996) and Baumgardt et al. (2005). Whereas the derived declinations of all clusters agree with the literature values within $\pm 3^{\prime \prime}$, the right ascensions show larger deviations: $4^{\prime \prime}$ for AM 1, 7" for Pal 3, and most significantly $62^{\prime \prime}$ for Pal 14. The deviating right ascension of Pal 14 goes back to the list of Webbink (1985), and then was taken over into the lists of Djorgovski \& Meylan (1993) and Harris (1996).

Concerning ellipticities, none of the clusters shows a measurable signal. They all seem to be relaxed, roundish halo clusters. Note however that a wide field study of main sequence stars around $\mathrm{Pal} 3$ revealed a weak signature of extra tidal member stars between one and four tidal radii (Sohn et al. 2003).

The half-light radii of the clusters were estimated from curve-of-growth analyses of the integrated light of the probable member stars. Our derived values deviate by $\pm 0.2^{\prime}$ from those given in Harris' list which are based on CCD work by Trager et al. (1993) and van den Bergh et al. (1991). Biases in the different selection of member stars and the accidental inclusion of foreground stars might explain these differences.
The core and tidal radii of the three clusters were derived from King profile fits to their surface number density as well as surface brightness profiles. For AM 1 and Pal 3 both radii could be determined with stable solutions. For Pal 14, the spatial extension of the member stars was not sufficient to get a stable fit for the tidal radius. It was fixed to $5^{\prime}$, the mean value of Harris (1996) and Baumgardt et al. (2005). The core and tidal radius of Pal 14 in Harris' list is based on the photographic study by Harris \& van den Bergh (1984), the ones of AM 1 and Pal 3 on the CCD work by Trager et al. (1993). The main differences of our findings to previous literature values are that AM 1 definitely has a larger tidal radius than claimed by Trager et al. (1993), but smaller than calculated by Baumgardt et al. (2005). On the contrary, the tidal radius of Pal 3 seems to be smaller than claimed by the two authors. The core radii of all clusters agree more or less with those given in Harris' list. The three clusters have in common that their central concentration parameter is around 1.0, very low in comparison to ordinary inner halo globular clusters.

This paper provides the basis for further investigations of evolved stars in AM 1, Pal 3, and Pal 14. Future follow-up spectroscopic observations of these cluster are needed. The measurements of a low (Newtonian) velocity dispersion would mean that MOND in its present form is not appropriate and that globular clusters do not possess dark matter. In contrast, a high velocity dispersion would either favour MOND or could be a hint to the existence of dark matter in globular clusters, a highly interesting hypothesis.

Acknowledgements. I wish to thank Holger Baumgardt and Klaas de Boer for very helpful comments, and the anonymous referee for useful suggestions which improved the paper. This project was supported by the DFG project HI 855/2.

\section{Appendix A: Lists of probable member stars}

The following tables contain the lists of probable member stars of AM 1, Pal3, and Pal 14. They are ordered by increasing $V$ magnitude. The columns are as follows:

Column 1. Identification number of the object, following the order of increasing $V$ magnitude.

Column 2. Right ascension for the epoch 2000 in hours, minutes and seconds $\left(\mathrm{h}, \mathrm{m} \mathrm{s}^{\mathrm{s}}\right)$.

Column 3. Declination (2000) in degrees, minutes and seconds $\left({ }^{\circ},{ }^{\prime},{ }^{\prime \prime}\right)$.

The positions of all objects were determined relative to positions in the USNO catalog. The positional accuracy of the calculated coordinates is in all fields better than $0.2^{\prime \prime}$.

Column 4. $V$ apparent magnitude as determined by PSF photometry under DAOPHOT II.

Column 5. $B-V$ colours from PSF photometry.

Column 6. Photometric error in $V$ (ALLSTAR output).

Column 7. Photometric error in $B$ (ALLSTAR output).

Column 8. Projected distance to cluster centre (see Table 3) in arcminutes. 
Table A.1. List of probable member stars of AM 1, ordered by increasing $V$ magnitude.

\begin{tabular}{|c|c|c|c|c|c|c|c|c|}
\hline$\overline{\mathrm{Id}}$ & $\begin{array}{c}\alpha(2000) \\
{[\mathrm{h}: \mathrm{m}: \mathrm{s}]}\end{array}$ & $\begin{array}{c}\delta(2000) \\
{\left[{ }^{0}:^{\prime}:^{\prime \prime}\right]}\end{array}$ & $\begin{array}{c}V \\
{[\mathrm{mag}]}\end{array}$ & $\begin{array}{l}B-V \\
{[\mathrm{mag}]}\end{array}$ & $\begin{array}{c}\sigma_{V} \\
{[\mathrm{mag}]}\end{array}$ & $\begin{array}{c}\sigma_{B} \\
{[\mathrm{mag}]}\end{array}$ & $\begin{array}{c}\text { radius } \\
{\left[{ }^{\prime}\right]}\end{array}$ & Type \\
\hline 1 & $03: 55: 02.93$ & $-49: 36: 44.6$ & 18.27 & 1.45 & 0.01 & 0.01 & 0.21 & RGB \\
\hline 2 & 03:55:06.36 & $-49: 36: 09.5$ & 19.44 & 1.07 & 0.01 & 0.01 & 1.01 & RGB \\
\hline 3 & 03:55:00.64 & $-49: 37: 17.9$ & 19.48 & 1.06 & 0.01 & 0.01 & 0.46 & RGB \\
\hline 4 & 03:55:02.04 & $-49: 36: 51.7$ & 19.51 & 1.02 & 0.01 & 0.01 & 0.07 & RGB \\
\hline 5 & 03:55:06.33 & $-49: 36: 20.2$ & 19.97 & 0.95 & 0.01 & 0.01 & 0.88 & RGB \\
\hline 6 & 03:55:01.82 & $-49: 36: 39.9$ & 20.12 & 0.92 & 0.01 & 0.01 & 0.27 & RGB \\
\hline 7 & 03:55:02.15 & $-49: 36: 50.1$ & 20.37 & 0.90 & 0.01 & 0.02 & 0.09 & RGB \\
\hline 8 & 03:55:01.79 & $-49: 41: 06.0$ & 20.43 & 0.73 & 0.01 & 0.01 & 4.18 & AGB \\
\hline 9 & 03:55:04.19 & $-49: 36: 55.7$ & 20.45 & 0.67 & 0.01 & 0.01 & 0.31 & AGB \\
\hline 10 & 03:55:06.78 & $-49: 37: 02.1$ & 20.52 & 0.75 & 0.01 & 0.02 & 0.74 & AGB \\
\hline 11 & 03:55:01.31 & $-49: 36: 41.6$ & 20.62 & 0.81 & 0.01 & 0.02 & 0.28 & RGB \\
\hline 12 & 03:55:05.70 & $-49: 37: 25.9$ & 20.63 & 0.67 & 0.01 & 0.01 & 0.75 & AGB \\
\hline 13 & $03: 55: 03.25$ & $-49: 37: 04.3$ & 20.66 & 0.62 & 0.01 & 0.01 & 0.21 & $\mathrm{HB}$ \\
\hline 14 & 03:55:02.36 & $-49: 36: 56.9$ & 20.73 & 0.63 & 0.02 & 0.02 & 0.03 & HB \\
\hline 15 & 03:54:59.64 & $-49: 38: 34.8$ & 20.78 & 0.63 & 0.01 & 0.01 & 1.71 & HB \\
\hline 16 & 03:54:58.65 & $-49: 37: 39.3$ & 20.78 & 0.80 & 0.02 & 0.02 & 0.94 & RGB \\
\hline 17 & 03:55:02.50 & $-49: 37: 00.7$ & 20.80 & 0.65 & 0.03 & 0.04 & 0.09 & HB \\
\hline 18 & 03:54:59.63 & $-49: 36: 45.8$ & 20.82 & 0.80 & 0.01 & 0.02 & 0.46 & RGB \\
\hline 19 & 03:55:01.04 & $-49: 36: 47.6$ & 20.87 & 0.73 & 0.01 & 0.02 & 0.24 & RGB \\
\hline 20 & 03:54:59.02 & $-49: 36: 44.9$ & 20.87 & 0.79 & 0.01 & 0.02 & 0.56 & RGB \\
\hline 21 & 03:55:01.27 & $-49: 36: 45.5$ & 20.88 & 0.59 & 0.01 & 0.01 & 0.23 & $\mathrm{HB}$ \\
\hline 22 & 03:55:00.68 & $-49: 36: 45.0$ & 20.88 & 0.80 & 0.01 & 0.02 & 0.31 & RGB \\
\hline 23 & 03:55:04.07 & $-49: 37: 17.0$ & 20.88 & 0.64 & 0.02 & 0.02 & 0.46 & $\mathrm{HB}$ \\
\hline 24 & 03:55:02.75 & $-49: 38: 43.6$ & 20.89 & 0.59 & 0.01 & 0.01 & 1.80 & HB \\
\hline 25 & 03:55:01.93 & $-49: 36: 54.6$ & 20.89 & 0.52 & 0.02 & 0.02 & 0.06 & HB \\
\hline 26 & 03:54:59.91 & $-49: 38: 01.7$ & 20.89 & 0.82 & 0.01 & 0.02 & 1.17 & RGB \\
\hline 27 & 03:55:04.57 & $-49: 37: 02.1$ & 20.92 & 0.57 & 0.01 & 0.02 & 0.39 & HB \\
\hline 28 & 03:55:02.92 & $-49: 37: 00.0$ & 20.92 & 0.80 & 0.02 & 0.02 & 0.13 & RGB \\
\hline 29 & 03:54:59.82 & $-49: 36: 56.1$ & 20.92 & 0.54 & 0.01 & 0.02 & 0.40 & $\mathrm{HB}$ \\
\hline 30 & 03:55:03.96 & $-49: 36: 51.9$ & 20.93 & 0.54 & 0.01 & 0.02 & 0.28 & $\mathrm{HB}$ \\
\hline
\end{tabular}

Table A.2. List of probable member stars of Pal 3, ordered by increasing $V$ magnitude.

\begin{tabular}{|c|c|c|c|c|c|c|c|c|}
\hline Id & $\begin{array}{c}\alpha(2000) \\
{[\mathrm{h}: \mathrm{m}: \mathrm{s}]}\end{array}$ & $\begin{array}{c}\delta(2000) \\
{\left[:^{0}::^{\prime \prime}\right]}\end{array}$ & $\begin{array}{c}V \\
{[\mathrm{mag}]}\end{array}$ & $\begin{array}{l}B-V \\
{[\mathrm{mag}]}\end{array}$ & $\begin{array}{c}\sigma_{V} \\
{[\mathrm{mag}]}\end{array}$ & $\begin{array}{c}\sigma_{B} \\
{[\mathrm{mag}]}\end{array}$ & $\begin{array}{c}\text { radius } \\
{\left[{ }^{\prime}\right]}\end{array}$ & Type \\
\hline 1 & $10: 05: 36.24$ & 00:03:59.9 & 18.05 & 1.16 & 0.01 & 0.01 & 1.11 & RGB \\
\hline 2 & $10: 05: 31.57$ & 00:04:17.0 & 18.25 & 1.13 & 0.02 & 0.01 & 0.10 & RGB \\
\hline 3 & 10:05:37.08 & 00:04:27.9 & 18.37 & 1.12 & 0.01 & 0.01 & 1.29 & RGB \\
\hline 4 & $10: 05: 29.71$ & 00:05:39.2 & 18.64 & 0.95 & 0.02 & 0.01 & 1.46 & AGB? \\
\hline 5 & 10:05:31.05 & 00:04:17.3 & 18.76 & 1.00 & 0.01 & 0.01 & 0.23 & RGB \\
\hline 6 & $10: 05: 34.63$ & 00:04:06.9 & 19.30 & 0.89 & 0.01 & 0.01 & 0.69 & RGB \\
\hline 7 & $10: 05: 21.69$ & 00:07:35.2 & 19.30 & 0.81 & 0.02 & 0.01 & 4.17 & AGB? \\
\hline 8 & $10: 05: 31.84$ & 00:04:16.5 & 19.34 & 0.86 & 0.02 & 0.01 & 0.04 & RGB \\
\hline 9 & $10: 05: 32.87$ & 00:04:36.1 & 19.37 & 0.88 & 0.02 & 0.01 & 0.38 & RGB \\
\hline 10 & 10:05:33.19 & 00:03:57.1 & 19.42 & 0.88 & 0.02 & 0.01 & 0.47 & RGB \\
\hline 11 & $10: 05: 27.72$ & 00:05:08.8 & 19.54 & 0.85 & 0.02 & 0.01 & 1.35 & RGB \\
\hline 12 & $10: 05: 31.12$ & 00:04:16.9 & 19.59 & 0.89 & 0.02 & 0.01 & 0.21 & RGB \\
\hline 13 & 10:05:32.91 & 00:04:26.1 & 19.80 & 0.85 & 0.02 & 0.01 & 0.27 & RGB \\
\hline 14 & 10:05:32.59 & 00:04:32.3 & 19.85 & 0.82 & 0.02 & 0.01 & 0.29 & RGB \\
\hline 15 & $10: 05: 32.73$ & $00: 04: 36.7$ & 19.97 & 0.81 & 0.02 & 0.01 & 0.37 & RGB \\
\hline 16 & 10:05:29.92 & 00:04:53.9 & 20.04 & 0.77 & 0.04 & 0.03 & 0.78 & RGB \\
\hline 17 & $10: 05: 28.29$ & $00: 04: 37.7$ & 20.08 & 0.61 & 0.01 & 0.01 & 0.97 & RGB \\
\hline 18 & 10:05:23.96 & $00: 04: 24.5$ & 20.12 & 0.71 & 0.01 & 0.01 & 2.00 & AGB \\
\hline 19 & $10: 05: 30.25$ & 00:03:37.6 & 20.14 & 0.80 & 0.01 & 0.01 & 0.80 & RGB \\
\hline 20 & $10: 05: 30.36$ & 00:05:29.6 & 20.18 & 0.59 & 0.02 & 0.01 & 1.26 & AGB \\
\hline 21 & $10: 05: 28.83$ & $00: 04: 36.5$ & 20.22 & 0.79 & 0.01 & 0.01 & 0.84 & RGB \\
\hline 22 & $10: 05: 28.79$ & 00:03:45.5 & 20.22 & 0.78 & 0.01 & 0.01 & 0.96 & RGB \\
\hline 23 & $10: 05: 29.97$ & 00:04:03.8 & 20.26 & 0.78 & 0.01 & 0.01 & 0.55 & RGB \\
\hline 24 & $10: 05: 30.44$ & 00:03:38.1 & 20.27 & 0.28 & 0.01 & 0.01 & 0.77 & $\mathrm{HB}$ \\
\hline 25 & $10: 05: 34.35$ & $00: 04: 39.2$ & 20.27 & 0.65 & 0.01 & 0.01 & 0.70 & AGB \\
\hline 26 & $10: 05: 33.82$ & 00:04:18.2 & 20.28 & 0.34 & 0.02 & 0.01 & 0.47 & HB \\
\hline 27 & $10: 05: 21.94$ & 00:04:06.3 & 20.30 & 0.33 & 0.02 & 0.01 & 2.51 & $\mathrm{HB}$ \\
\hline 28 & $10: 05: 30.07$ & 00:02:04.7 & 20.31 & 0.80 & 0.02 & 0.01 & 2.27 & RGB \\
\hline 29 & $10: 05: 31.49$ & $00: 04: 27.7$ & 20.31 & 0.76 & 0.02 & 0.01 & 0.20 & RGB \\
\hline 30 & $10: 05: 31.35$ & 00:04:14.4 & 20.33 & 0.28 & 0.02 & 0.01 & 0.16 & $\mathrm{HB}$ \\
\hline
\end{tabular}

Table A.3. List of probable member stars of Pal 14, ordered by increasing $V$ magnitude.

\begin{tabular}{|c|c|c|c|c|c|c|c|c|}
\hline Id & $\begin{array}{c}\alpha(2000) \\
{[\mathrm{h}: \mathrm{m}: \mathrm{s}]}\end{array}$ & $\begin{array}{c}\delta(2000) \\
{\left[{ }^{\circ}:^{\prime}:^{\prime \prime}\right]} \\
\end{array}$ & $\begin{array}{c}V \\
{[\mathrm{mag}]} \\
\end{array}$ & $\begin{array}{l}B-V \\
{[\mathrm{mag}]}\end{array}$ & $\begin{array}{c}\sigma_{V} \\
{[\mathrm{mag}]}\end{array}$ & $\begin{array}{c}\sigma_{B} \\
{[\mathrm{mag}]}\end{array}$ & $\begin{array}{c}\text { radius } \\
{\left[{ }^{\prime}\right]} \\
\end{array}$ & Type \\
\hline 1 & $16: 11: 05.81$ & $14: 57: 45.1$ & 17.37 & 1.39 & 0.02 & 0.01 & 1.28 & RGB \\
\hline 2 & $16: 10: 58.73$ & $14: 56: 48.7$ & 17.77 & 1.29 & 0.02 & 0.01 & 0.80 & RGB \\
\hline 3 & $16: 10: 54.90$ & $14: 58: 36.7$ & 18.23 & 1.07 & 0.01 & 0.01 & 1.80 & AGB? \\
\hline 4 & $16: 11: 04.98$ & $14: 53: 32.3$ & 18.48 & 1.10 & 0.02 & 0.01 & 4.07 & RGB \\
\hline 5 & $16: 10: 59.24$ & $14: 57: 22.5$ & 18.52 & 0.99 & 0.01 & 0.01 & 0.35 & AGB? \\
\hline 6 & $16: 10: 53.36$ & $14: 56: 45.4$ & 18.56 & 1.04 & 0.01 & 0.01 & 1.90 & RGB \\
\hline 7 & $16: 10: 54.04$ & $14: 57: 05.6$ & 18.70 & 1.03 & 0.01 & 0.01 & 1.64 & RGB \\
\hline 8 & $16: 10: 56.90$ & $14: 57: 56.5$ & 18.84 & 0.99 & 0.01 & 0.01 & 1.02 & RGB \\
\hline 9 & $16: 11: 01.40$ & $14: 57: 60.0$ & 19.05 & 0.99 & 0.01 & 0.01 & 0.56 & RGB \\
\hline 10 & $16: 11: 05.89$ & $14: 58: 43.2$ & 19.19 & 0.93 & 0.02 & 0.01 & 1.78 & RGB \\
\hline 11 & $16: 11: 06.00$ & $14: 55: 39.1$ & 19.37 & 0.95 & 0.01 & 0.01 & 2.23 & RGB \\
\hline 12 & $16: 10: 56.21$ & $14: 56: 32.7$ & 19.41 & 0.90 & 0.01 & 0.01 & 1.42 & RGB \\
\hline 13 & $16: 10: 56.98$ & $14: 56: 25.8$ & 19.44 & 0.92 & 0.01 & 0.01 & 1.37 & RGB \\
\hline 14 & $16: 10: 59.24$ & $14: 57: 19.7$ & 19.50 & 0.90 & 0.02 & 0.01 & 0.37 & RGB \\
\hline 15 & $16: 10: 55.84$ & $14: 57: 43.4$ & 19.60 & 0.69 & 0.01 & 0.01 & 1.19 & AGB \\
\hline 16 & $16: 10: 59.62$ & $15: 01: 32.9$ & 19.68 & 0.81 & 0.02 & 0.01 & 4.09 & RGB \\
\hline 17 & $16: 11: 00.58$ & $14: 56: 59.1$ & 19.76 & 0.86 & 0.01 & 0.01 & 0.48 & RGB \\
\hline 18 & $16: 11: 03.91$ & $14: 56: 34.3$ & 19.77 & 0.59 & 0.01 & 0.01 & 1.19 & AGB \\
\hline 19 & 16:11:01.01 & $14: 55: 23.1$ & 19.78 & 0.69 & 0.01 & 0.01 & 2.09 & AGB \\
\hline 20 & $16: 11: 07.42$ & $14: 57: 43.2$ & 19.86 & 0.86 & 0.01 & 0.01 & 1.65 & RGB \\
\hline 21 & $16: 10: 52.81$ & $14: 55: 38.9$ & 19.87 & 0.84 & 0.01 & 0.01 & 2.62 & RGB \\
\hline 22 & $16: 10: 56.17$ & $14: 55: 48.1$ & 19.88 & 0.46 & 0.01 & 0.01 & 1.99 & HB \\
\hline 23 & $16: 11: 02.52$ & $14: 56: 49.7$ & 19.88 & 0.62 & 0.01 & 0.01 & 0.78 & HB \\
\hline 24 & 16:11:10.09 & $14: 55: 54.9$ & 19.89 & 0.88 & 0.02 & 0.01 & 2.76 & RGB \\
\hline 25 & $16: 11: 04.45$ & $14: 56: 14.4$ & 19.90 & 0.65 & 0.01 & 0.01 & 1.53 & HB \\
\hline 26 & $16: 10: 59.32$ & $14: 57: 25.9$ & 19.92 & 0.84 & 0.02 & 0.01 & 0.32 & RGB \\
\hline 27 & $16: 11: 16.22$ & 15:00:07.1 & 19.92 & 0.47 & 0.02 & 0.01 & 4.60 & HB \\
\hline 28 & 16:10:59.89 & $14: 53: 27.8$ & 19.92 & 0.92 & 0.03 & 0.02 & 4.01 & RGB \\
\hline 29 & $16: 11: 12.60$ & $14: 56: 55.2$ & 19.93 & 0.46 & 0.02 & 0.01 & 2.94 & $\mathrm{HB}$ \\
\hline 30 & $16: 11: 13.56$ & $14: 57: 58.2$ & 19.95 & 0.85 & 0.02 & 0.01 & 3.16 & RGB \\
\hline
\end{tabular}

Column 9. "Type" describes to which part of the CMD the star probably belongs: $\mathrm{RGB}=$ red giant branch, $\mathrm{HB}=$ horizontal branch, AGB = asymptotic giant branch.

Note: Here we present only the 30 brightest stars of each cluster. The full tables with the magnitude limited samples of probable member stars (see Sect. 4.1) are only available in the online version of the article.

\section{References}

Armandroff, T. E., Da Costa, G. S., \& Zinn, R. 1992, AJ, 104, 164 Baumgardt, H., Grebel, E. K., \& Kroupa, P. 2005, MNRAS, 359, L1 Bekenstein, J., \& Milgrom, M. 1984, ApJ, 286, 7

Catelan, M., Ferraro, F. R., \& Rood, R. T. 2001, ApJ, 560, 970

Djorgovsky, S., \& Meylan, G. 1993, in Structure and Dynamics of Globular Clusters, ed. G. Meylan, \& S. Djorgovski (San Francisco: ASP), ASP Conf. Ser., 50, 325

Harris, W. E. 1996, AJ, 112, 1487

Harris, W. E., \& van den Bergh, S. 1984, AJ, 89, 1816

Holland, S., \& Harris, W. E. 1992, AJ, 103, 131

Kim, Y., Demarque, P., Yi, S. K., \& Alexander, D. R. 2002, ApJS, 143, 499

King, I. 1962, AJ, 67, 471

Mackey, A. D., \& Gilmore, G. F. 2004, MNRAS, 355, 504

Mackey, A. D., \& van den Bergh, S. 2005, MNRAS, 360, 631 
Madore, B. F., \& Freedman, W. L. 1989, ApJ, 340, 812

Mashchenko, S., \& Sills, A. 2005, ApJ, 619, 243

Milgrom, M. 1983, ApJ, 270, 365

Ortolani, S., \& Gratton, R. G. 1989, A\&AS, 79, 155

Sarajedini, A. 1997, AJ, 113, 682

Schlegel, D. J., Finkbeiner, D. P., \& Davis, M. 1998, ApJ, 500, 525

Searle, L., \& Zinn, R. 1978, ApJ, 225, 357

Sohn, Y.-J., Park, J.-H., Rey, S.-C., et al. 2003, AJ, 126, 803

Stetson, P. B. 1987, PASP, 99, 191

Stetson, P. B. 1992, in Astronomical Data Analysis Software and Systems I, ed. D. M. Worrall, C. Biemesderfer, \& J. Barnes (San Francisco: ASP), ASP Conf. Ser., 25, 297

Stetson, P. B., Bolte, M., Harris, W. E., et al. 1999, AJ, 117, 247
Suntzeff, N., Olszewski, E., \& Stetson, P. B. 1985, AJ, 90, 1481

Trager, S. C., Djorgovski, S., \& King, I. R. 1993, in Structure and Dynamics of Globular Clusters, ed. S. G. Djorgovski, \& G. Meylan (San Francisco: ASP), ASP Conf. Ser., 50, 347

van den Berg, D. A. 2000, ApJS, 129, 315

van den Bergh, S., \& Mackey, A. D. 2004, MNRAS, 354, 713

van den Bergh, S., Morbey, C., \& Pazder, J. 1991, ApJ, 375, 594

Webbink, R. F. 1985, in Dynamics of Star Clusters, ed. J. Goodman, \& P. Hut (Dordrecht: Reidel), IAU Symp., 113, 541

Zinn, R. 1985, ApJ, 293, 424

Zinn, R. 1993, in The Globular Cluster-Galaxy Connection, ed. G. H. Smith, \& J. P. Brodie, (San Francisco: ASP), ASP Conf. Ser., 48, 38 


\section{Online Material}


M. Hilker: Probable member stars of AM 1, Pal 3 and Pal 14, Online Material p 2

Table A.1. List of probable member stars of AM 1, ordered by increasing $V$ magnitude.

\begin{tabular}{|c|c|c|c|c|c|c|c|c|}
\hline Id & $\begin{array}{c}\alpha(2000) \\
{[\mathrm{h}: \mathrm{m}: \mathrm{s}]}\end{array}$ & $\begin{array}{c}\delta(2000) \\
{\left[{ }^{\circ}:^{\prime}:^{\prime \prime}\right]}\end{array}$ & $\begin{array}{c}V \\
{[\mathrm{mag}]}\end{array}$ & $\begin{array}{l}B-V \\
{[\mathrm{mag}]}\end{array}$ & $\begin{array}{c}\sigma_{V} \\
{[\mathrm{mag}]}\end{array}$ & $\begin{array}{c}\sigma_{B} \\
{[\mathrm{mag}]}\end{array}$ & $\begin{array}{c}\text { radius } \\
{\left[{ }^{\prime}\right]}\end{array}$ & Type \\
\hline 1 & $03: 55: 02.93$ & $-49: 36: 44.6$ & 18.27 & 1.45 & 0.01 & 0.01 & 0.21 & RGB \\
\hline 2 & 03:55:06.36 & $-49: 36: 09.5$ & 19.44 & 1.07 & 0.01 & 0.01 & 1.01 & RGB \\
\hline 3 & 03:55:00.64 & $-49: 37: 17.9$ & 19.48 & 1.06 & 0.01 & 0.01 & 0.46 & RGB \\
\hline 4 & 03:55:02.04 & $-49: 36: 51.7$ & 19.51 & 1.02 & 0.01 & 0.01 & 0.07 & RGB \\
\hline 5 & $03: 55: 06.33$ & $-49: 36: 20.2$ & 19.97 & 0.95 & 0.01 & 0.01 & 0.88 & RGB \\
\hline 6 & 03:55:01.82 & $-49: 36: 39.9$ & 20.12 & 0.92 & 0.01 & 0.01 & 0.27 & RGB \\
\hline 7 & 03:55:02.15 & $-49: 36: 50.1$ & 20.37 & 0.90 & 0.01 & 0.02 & 0.09 & RGB \\
\hline 8 & 03:55:01.79 & $-49: 41: 06.0$ & 20.43 & 0.73 & 0.01 & 0.01 & 4.18 & AGB \\
\hline 9 & $03: 55: 04.19$ & $-49: 36: 55.7$ & 20.45 & 0.67 & 0.01 & 0.01 & 0.31 & AGB \\
\hline 10 & 03:55:06.78 & $-49: 37: 02.1$ & 20.52 & 0.75 & 0.01 & 0.02 & 0.74 & AGB \\
\hline 11 & 03:55:01.31 & $-49: 36: 41.6$ & 20.62 & 0.81 & 0.01 & 0.02 & 0.28 & RGB \\
\hline 12 & 03:55:05.70 & $-49: 37: 25.9$ & 20.63 & 0.67 & 0.01 & 0.01 & 0.75 & AGB \\
\hline 13 & 03:55:03.25 & $-49: 37: 04.3$ & 20.66 & 0.62 & 0.01 & 0.01 & 0.21 & $\mathrm{HB}$ \\
\hline 14 & $03: 55: 02.36$ & $-49: 36: 56.9$ & 20.73 & 0.63 & 0.02 & 0.02 & 0.03 & $\mathrm{HB}$ \\
\hline 15 & $03: 54: 59.64$ & $-49: 38: 34.8$ & 20.78 & 0.63 & 0.01 & 0.01 & 1.71 & $\mathrm{HB}$ \\
\hline 16 & 03:54:58.65 & $-49: 37: 39.3$ & 20.78 & 0.80 & 0.02 & 0.02 & 0.94 & RGB \\
\hline 17 & $03: 55: 02.50$ & $-49: 37: 00.7$ & 20.80 & 0.65 & 0.03 & 0.04 & 0.09 & $\mathrm{HB}$ \\
\hline 18 & 03:54:59.63 & $-49: 36: 45.8$ & 20.82 & 0.80 & 0.01 & 0.02 & 0.46 & RGB \\
\hline 19 & 03:55:01.04 & $-49: 36: 47.6$ & 20.87 & 0.73 & 0.01 & 0.02 & 0.24 & RGB \\
\hline 20 & 03:54:59.02 & $-49: 36: 44.9$ & 20.87 & 0.79 & 0.01 & 0.02 & 0.56 & RGB \\
\hline 21 & 03:55:01.27 & $-49: 36: 45.5$ & 20.88 & 0.59 & 0.01 & 0.01 & 0.23 & $\mathrm{HB}$ \\
\hline 22 & 03:55:00.68 & $-49: 36: 45.0$ & 20.88 & 0.80 & 0.01 & 0.02 & 0.31 & RGB \\
\hline 23 & 03:55:04.07 & $-49: 37: 17.0$ & 20.88 & 0.64 & 0.02 & 0.02 & 0.46 & $\mathrm{HB}$ \\
\hline 24 & 03:55:02.75 & $-49: 38: 43.6$ & 20.89 & 0.59 & 0.01 & 0.01 & 1.80 & $\mathrm{HB}$ \\
\hline 25 & 03:55:01.93 & $-49: 36: 54.6$ & 20.89 & 0.52 & 0.02 & 0.02 & 0.06 & HB \\
\hline 26 & 03:54:59.91 & $-49: 38: 01.7$ & 20.89 & 0.82 & 0.01 & 0.02 & 1.17 & RGB \\
\hline 27 & $03: 55: 04.57$ & $-49: 37: 02.1$ & 20.92 & 0.57 & 0.01 & 0.02 & 0.39 & $\mathrm{HB}$ \\
\hline 28 & 03:55:02.92 & $-49: 37: 00.0$ & 20.92 & 0.80 & 0.02 & 0.02 & 0.13 & RGB \\
\hline 29 & 03:54:59.82 & $-49: 36: 56.1$ & 20.92 & 0.54 & 0.01 & 0.02 & 0.40 & $\mathrm{HB}$ \\
\hline 30 & 03:55:03.96 & $-49: 36: 51.9$ & 20.93 & 0.54 & 0.01 & 0.02 & 0.28 & HB \\
\hline 31 & 03:55:03.63 & $-49: 36: 45.1$ & 20.94 & 0.56 & 0.02 & 0.02 & 0.28 & $\mathrm{HB}$ \\
\hline 32 & 03:55:02.85 & $-49: 36: 49.7$ & 20.94 & 0.64 & 0.02 & 0.02 & 0.13 & HB \\
\hline 33 & 03:55:02.65 & $-49: 36: 44.0$ & 20.94 & 0.53 & 0.02 & 0.02 & 0.20 & $\mathrm{HB}$ \\
\hline 34 & $03: 54: 57.27$ & $-49: 36: 08.0$ & 20.96 & 0.57 & 0.01 & 0.01 & 1.13 & HB \\
\hline 35 & $03: 54: 59.62$ & $-49: 36: 40.7$ & 20.96 & 0.54 & 0.01 & 0.01 & 0.50 & $\mathrm{HB}$ \\
\hline 36 & 03:55:01.82 & $-49: 37: 14.8$ & 20.98 & 0.55 & 0.02 & 0.02 & 0.33 & $\mathrm{HB}$ \\
\hline 37 & 03:54:43.93 & $-49: 34: 34.2$ & 20.98 & 0.75 & 0.02 & 0.02 & 3.80 & RGB \\
\hline 38 & 03:55:02.13 & $-49: 37: 26.1$ & 20.98 & 0.60 & 0.01 & 0.02 & 0.51 & HB \\
\hline 39 & 03:55:01.19 & $-49: 36: 30.6$ & 20.98 & 0.58 & 0.01 & 0.02 & 0.45 & $\mathrm{HB}$ \\
\hline 40 & 03:54:58.67 & $-49: 36: 20.8$ & 20.98 & 0.52 & 0.01 & 0.01 & 0.82 & HB \\
\hline 41 & 03:55:03.07 & $-49: 36: 46.5$ & 20.99 & 0.59 & 0.02 & 0.02 & 0.19 & $\mathrm{HB}$ \\
\hline 42 & 03:55:03.54 & $-49: 36: 43.9$ & 20.99 & 0.53 & 0.02 & 0.02 & 0.28 & HB \\
\hline 43 & 03:55:01.39 & $-49: 36: 47.4$ & 20.99 & 0.57 & 0.01 & 0.02 & 0.20 & HB \\
\hline 44 & $03: 54: 50.84$ & $-49: 38: 21.4$ & 21.00 & 0.64 & 0.01 & 0.02 & 2.34 & HB \\
\hline 45 & 03:55:04.06 & $-49: 37: 01.5$ & 21.00 & 0.52 & 0.02 & 0.02 & 0.30 & $\mathrm{HB}$ \\
\hline 46 & 03:55:04.85 & $-49: 36: 48.4$ & 21.00 & 0.54 & 0.01 & 0.02 & 0.43 & HB \\
\hline 47 & 03:55:06.02 & $-49: 37: 05.8$ & 21.01 & 0.55 & 0.01 & 0.01 & 0.63 & $\mathrm{HB}$ \\
\hline 48 & 03:55:00.81 & $-49: 37: 01.1$ & 21.01 & 0.55 & 0.01 & 0.02 & 0.26 & HB \\
\hline 49 & 03:55:01.38 & $-49: 37: 00.4$ & 21.02 & 0.52 & 0.02 & 0.02 & 0.17 & $\mathrm{HB}$ \\
\hline 50 & 03:55:02.01 & $-49: 36: 55.6$ & 21.02 & 0.53 & 0.02 & 0.02 & 0.05 & HB \\
\hline 51 & 03:55:04.22 & $-49: 37: 04.0$ & 21.02 & 0.79 & 0.02 & 0.02 & 0.34 & RGB \\
\hline 52 & 03:55:00.59 & $-49: 36: 50.9$ & 21.02 & 0.55 & 0.02 & 0.02 & 0.29 & HB \\
\hline 53 & $03: 54: 59.20$ & $-49: 36: 10.3$ & 21.02 & 0.53 & 0.02 & 0.01 & 0.90 & $\mathrm{HB}$ \\
\hline 54 & 03:55:01.43 & $-49: 36: 35.8$ & 21.02 & 0.52 & 0.02 & 0.02 & 0.36 & HB \\
\hline 55 & 03:55:02.35 & $-49: 36: 55.9$ & 21.03 & 0.72 & 0.03 & 0.03 & 0.01 & RGB \\
\hline 56 & 03:55:02.30 & $-49: 36: 37.9$ & 21.04 & 0.56 & 0.01 & 0.02 & 0.29 & HB \\
\hline 57 & 03:55:01.43 & $-49: 37: 05.7$ & 21.04 & 0.77 & 0.01 & 0.02 & 0.22 & RGB \\
\hline 58 & 03:55:07.14 & $-49: 36: 39.7$ & 21.04 & 0.76 & 0.01 & 0.02 & 0.83 & RGB \\
\hline 59 & 03:54:59.93 & $-49: 36: 51.5$ & 21.06 & 0.49 & 0.02 & 0.02 & 0.39 & $\mathrm{HB}$ \\
\hline
\end{tabular}

Table A.1. continued.

\begin{tabular}{|c|c|c|c|c|c|c|c|c|}
\hline Id & $\begin{array}{c}\alpha(2000) \\
{[\mathrm{h}: \mathrm{m}: \mathrm{s}]}\end{array}$ & $\begin{array}{c}\delta(2000) \\
{\left[{ }^{\circ}:^{\prime}:^{\prime \prime}\right]}\end{array}$ & $\begin{array}{c}V \\
{[\mathrm{mag}]}\end{array}$ & $\begin{array}{l}B-V \\
{[\mathrm{mag}]}\end{array}$ & $\begin{array}{c}\sigma_{V} \\
{[\mathrm{mag}]}\end{array}$ & $\begin{array}{c}\sigma_{B} \\
{[\mathrm{mag}]}\end{array}$ & $\begin{array}{c}\text { radius } \\
{\left[{ }^{\prime}\right]}\end{array}$ & Ty \\
\hline 60 & $3: 55: 03.35$ & $-49: 37: 12.9$ & 21.09 & 0.53 & 0.02 & 0.02 & 0.34 & $\mathrm{HB}$ \\
\hline 61 & 03:55:01.28 & $-49: 37: 06.2$ & 21.17 & 0.75 & 0.02 & 0.03 & 0.24 & RGB \\
\hline 62 & $03: 54$ & $-49: 3$ & 21.17 & 0.71 & 0.0 & 0.02 & 1.47 & RGB \\
\hline 63 & 03:55:02.59 & $-49: 37: 02.2$ & 21.17 & 0.77 & 0.02 & 0.04 & 0.12 & RGB \\
\hline 64 & $3: 55: 03.77$ & $-49: 38: 03.4$ & 21.30 & 0.41 & 0.02 & 0.02 & 1.16 & HB \\
\hline 65 & 03:55:02.97 & $-49: 37: 13.1$ & 21.31 & 0.68 & 0.03 & 0.02 & 0.31 & RGB \\
\hline 66 & 03:55:01.21 & $-49: 36: 20.9$ & 21.33 & 0.73 & 0.01 & 0.02 & 0.60 & RGB \\
\hline 67 & 03:55:02.67 & $-49: 36: 46.7$ & 21.37 & 0.69 & 0.02 & 0.03 & 0.16 & RGB \\
\hline 68 & 03:55:04.21 & $-49: 37: 29.2$ & 21.40 & 0.73 & 0.02 & 0.03 & 0.64 & RGB \\
\hline 69 & $3: 55: 03.66$ & $-49: 3 c$ & 21.41 & 0.79 & 0.02 & 0.03 & 0.25 & RGB \\
\hline 70 & $03: 55: 12.00$ & $-49: 34$ & 21.46 & .68 & 33 & .03 & 2.60 & RGB \\
\hline 71 & 03:55:01.91 & $-49: 3$ & 21.47 & 0.73 & 0.02 & 0.03 & 0.47 & RGB \\
\hline 72 & $03: 5$ & $-49: 37$ & 21.50 & 0.43 & 0.02 & 0.02 & 1.40 & HB \\
\hline 73 & 03:55:01.58 & $-49: 36: 56.1$ & 21.58 & 0.73 & 0.03 & 0.03 & 0.12 & RGB \\
\hline 74 & $03: 5$ & $-49: 36$ & 21.67 & 0.76 & 0.02 & 0.02 & 0.87 & RGB \\
\hline 75 & 03:55:0 & $-49: 3$ & 21.69 & 0.67 & 33 & 94 & 0.16 & RGB \\
\hline 76 & $03: 55: 03.23$ & $-49: 36: 51.6$ & 21.73 & 0.70 & 0.04 & 0.04 & 0.16 & RGB \\
\hline 77 & 03:55:06.81 & $-49: 37: 38.8$ & 21.85 & 0.66 & 0.03 & 0.03 & 1.03 & RGB \\
\hline 78 & 03:55:02.10 & $-49: 37: 21.2$ & 21.85 & 0.75 & 0.03 & 0.04 & 0.43 & RGB \\
\hline 79 & $03: 5$ & $-49: 3$ & 21.87 & 0.70 & 0.08 & 0.07 & 0.21 & RGB \\
\hline 80 & 1 & $-49: 3$ & 21.88 & 0.7 & & & .16 & RGB \\
\hline 81 & 03:55:02.31 & $-49: 37: 02.3$ & 21.91 & 0.77 & 0.03 & 0.05 & 0.11 & RGB \\
\hline 82 & $03: 54: 5$ & $-49: 34: 53.5$ & 21.95 & .72 & 0.03 & 4 & 2.81 & RGB \\
\hline 83 & 03:55:03.82 & $-49: 3$ & 22.04 & 0. & 0.03 & 0.04 & 0.45 & RGB \\
\hline 84 & $03: 5$ & $-49: 3$ & 22.06 & 0.68 & 0.03 & 0.04 & 0.39 & RGB \\
\hline 85 & & $-49: 3$ & 22.08 & 0.66 & 0.03 & 5 & 0.52 & RGB \\
\hline 86 & 03:55: & $-49: 37: 18.5$ & 22.14 & 0.71 & 0.04 & 0.04 & 0.39 & RGB \\
\hline 87 & $03: 55$ : & $-49: 36: 38.6$ & 22.17 & 0.65 & 0.03 & 4 & 0.43 & RGB \\
\hline 88 & $03: 5$ & -49 : & 22.22 & 0 & 0.03 & 0.04 & 0.32 & RGB \\
\hline 89 & 03.5 & $-49: ?$ & 22.33 & 2 & 0.03 & 0.04 & 1.15 & RGB \\
\hline 90 & $03: 5$ & $-49: 3$ & 22.34 & 0.65 & 0.04 & 0.05 & 0.82 & RGB \\
\hline 91 & $03: 55$ & $-49: 3$ & 22.35 & 0.68 & 0.04 & 0.06 & 0.23 & RGB \\
\hline 92 & $03: 5$ & $-49: 3$ & 22.36 & 0.64 & 0.04 & 5 & 0.90 & RGB \\
\hline 93 & 03:54: & $-49: 3$ & 22.39 & & 0.06 & 0.05 & 1.96 & RGB \\
\hline 94 & $03: 5$ & $-49: 3$ & 22.45 & 0.62 & 0.04 & 0.04 & 0.80 & RGB \\
\hline 95 & $03: 5$ & $-49: 36: 03.1$ & 22.47 & 0 . & 0.03 & 0.06 & 1.04 & RGB \\
\hline 96 & $03: 5$ & $-49: 3$ & 22.53 & 0.68 & 0.04 & 0.06 & 0.27 & RGB \\
\hline 97 & $03: 5$ & $-49: 40: 31.9$ & 22.56 & 0 & 0.06 & 6 & 3.86 & RGB \\
\hline 98 & 03:55:02.88 & $-49: 3$ & 22.58 & & 0.04 & & 0.40 & RGB \\
\hline 99 & $03: 5$ & $-49: 3$ & 22.60 & 0.63 & 0.06 & .07 & 0.26 & RGB \\
\hline 100 & $03: 5$ & $-49: 3$ & 22.61 & & 0.05 & 0.06 & 0.38 & RGB \\
\hline 101 & $03: 54$ & $-49: 37: 59.2$ & 22.61 & 0.73 & 0.05 & 0.07 & 3.15 & RGB \\
\hline 102 & $03: 54: 59.13$ & $-49: 37: 12.9$ & 22.67 & 0.63 & 0.06 & 0.07 & 0.59 & RGB \\
\hline 103 & $03: 55$ & $-49: 37: 09.1$ & 22.69 & 0.62 & 0.04 & 0.07 & 0.24 & RGB \\
\hline 104 & 03:55: & $-49: 36: 08.5$ & 22.69 & 0.63 & 0.05 & 0.07 & 3.30 & RGB \\
\hline 105 & 03:55:02.10 & $-49: 36: 45.8$ & 22.75 & 0.66 & 0.06 & 0.07 & 0.16 & RGB \\
\hline 106 & 03:54:49.76 & $-49: 37: 22.8$ & 22.77 & 0.59 & 0.07 & 0.06 & 2.08 & RGB \\
\hline 107 & 03:55:05.37 & $-49: 36: 44.6$ & 22.77 & 0.62 & 0.05 & 0.09 & 0.53 & RGB \\
\hline 108 & $03: 5$ & $-49: 37: 01.3$ & 22.78 & 0.60 & 0.05 & 0.10 & 0.10 & RGB \\
\hline 109 & 03:55:04.44 & $-49: 36: 37.5$ & 22.89 & 0.70 & 0.05 & 0.10 & 0.46 & RGB \\
\hline 110 & 03:55:01.53 & $-49: 37: 20.8$ & 22.91 & 0.67 & 0.06 & 0.08 & 0.44 & RGB \\
\hline
\end{tabular}


Table A.2. List of probable member stars of Pal3, ordered by increasing $V$ magnitude.

\begin{tabular}{|c|c|c|c|c|c|c|c|c|}
\hline Id & $\begin{array}{c}\alpha(2000) \\
{[\mathrm{h}: \mathrm{m}: \mathrm{s}]}\end{array}$ & $\begin{array}{c}\delta(2000) \\
{\left[{ }^{\circ}:^{\prime}:^{\prime \prime}\right]}\end{array}$ & $\begin{array}{c}V \\
{[\mathrm{mag}]}\end{array}$ & $\begin{array}{l}B-V \\
{[\mathrm{mag}]}\end{array}$ & $\begin{array}{c}\sigma_{V} \\
{[\mathrm{mag}]}\end{array}$ & $\begin{array}{c}\sigma_{B} \\
{[\mathrm{mag}]}\end{array}$ & $\begin{array}{c}\text { radius } \\
{\left[{ }^{\prime}\right]}\end{array}$ & Type \\
\hline 1 & $10: 05: 36.24$ & $00: 03: 59.9$ & 18.05 & 1.16 & 0.01 & 0.01 & 1.11 & RGB \\
\hline 2 & 10:05:31.57 & 00:04:17.0 & 18.25 & 1.13 & 0.02 & 0.01 & 0.10 & RGB \\
\hline 3 & 10:05:37.08 & $00: 04: 27.9$ & 18.37 & 1.12 & 0.01 & 0.01 & 1.29 & RGB \\
\hline 4 & 10:05:29.71 & $00: 05: 39.2$ & 18.64 & 0.95 & 0.02 & 0.01 & 1.46 & AGB? \\
\hline 5 & $10: 05: 31.05$ & $00: 04: 17.3$ & 18.76 & 1.00 & 0.01 & 0.01 & 0.23 & RGB \\
\hline 6 & $10: 05: 34.63$ & 00:04:06.9 & 19.30 & 0.89 & 0.01 & 0.01 & 0.69 & RGB \\
\hline 7 & $10: 05: 21.69$ & $00: 07: 35.2$ & 19.30 & 0.81 & 0.02 & 0.01 & 4.17 & AGB? \\
\hline 8 & $10: 05: 31.84$ & $00: 04: 16.5$ & 19.34 & 0.86 & 0.02 & 0.01 & 0.04 & RGB \\
\hline 9 & $10: 05: 32.87$ & 00:04:36.1 & 19.37 & 0.88 & 0.02 & 0.01 & 0.38 & RGB \\
\hline 10 & 10:05:33.19 & 00:03:57.1 & 19.42 & 0.88 & 0.02 & 0.01 & 0.47 & RGB \\
\hline 11 & $10: 05: 27.72$ & 00:05:08.8 & 19.54 & 0.85 & 0.02 & 0.01 & 1.35 & RGB \\
\hline 12 & $10: 05: 31.12$ & 00:04:16.9 & 19.59 & 0.89 & 0.02 & 0.01 & 0.21 & RGB \\
\hline 13 & 10:05:32.91 & $00: 04: 26.1$ & 19.80 & 0.85 & 0.02 & 0.01 & 0.27 & RGB \\
\hline 14 & $10: 05: 32.59$ & $00: 04: 32.3$ & 19.85 & 0.82 & 0.02 & 0.01 & 0.29 & RGB \\
\hline 15 & $10: 05: 32.73$ & $00: 04: 36.7$ & 19.97 & 0.81 & 0.02 & 0.01 & 0.37 & RGB \\
\hline 16 & 10:05:29.92 & 00:04:53.9 & 20.04 & 0.77 & 0.04 & 0.03 & 0.78 & RGB \\
\hline 17 & $10: 05: 28.29$ & $00: 04: 37.7$ & 20.08 & 0.61 & 0.01 & 0.01 & 0.97 & RGB \\
\hline 18 & $10: 05: 23.96$ & $00: 04: 24.5$ & 20.12 & 0.71 & 0.01 & 0.01 & 2.00 & AGB \\
\hline 19 & $10: 05: 30.25$ & $00: 03: 37.6$ & 20.14 & 0.80 & 0.01 & 0.01 & 0.80 & RGB \\
\hline 20 & $10: 05: 30.36$ & $00: 05: 29.6$ & 20.18 & 0.59 & 0.02 & 0.01 & 1.26 & AGB \\
\hline 21 & $10: 05: 28.83$ & $00: 04: 36.5$ & 20.22 & & 0.01 & & 0.84 & RGB \\
\hline 22 & $10: 05: 28.79$ & $00: 03: 45.5$ & 20.22 & 0.78 & 0.01 & 0.01 & 0.96 & RGB \\
\hline 23 & 10:05:29.97 & 00:04:03.8 & 20.26 & 0.78 & 0.01 & 0.01 & 0.55 & RGB \\
\hline 24 & $10: 0$ & $00: 03: 38.1$ & 20.27 & 0.28 & 0.01 & 0.01 & 0.77 & HB \\
\hline 25 & 10:05:34.35 & 00:04:39.2 & 20.27 & 0.65 & 0.01 & 0.01 & 0.70 & AGB \\
\hline 26 & $10: 05: 33.82$ & $00: 04: 18.2$ & 20.28 & 0.34 & 0.02 & 0.01 & 0.47 & $\mathrm{HB}$ \\
\hline 27 & $10: 05: 21.94$ & 00:04:06.3 & 20.30 & 0.33 & 0.02 & 0.01 & 2.51 & HB \\
\hline 28 & 10:05:30.07 & 00:02:04.7 & 20.31 & 0.80 & 0.02 & 0.01 & 2.27 & RGB \\
\hline 29 & $10: 05: 31.49$ & $00: 04: 27.7$ & 20.31 & 0.76 & 0.02 & 0.01 & 0.20 & RGB \\
\hline 30 & $10: 05: 31.35$ & $00: 04: 14.4$ & 20.33 & 0.28 & 0.02 & 0.01 & 0.16 & $\mathrm{HB}$ \\
\hline 31 & 10:05:35.79 & 00:03:34.3 & 20.33 & 0.50 & 0.01 & 0.01 & 1.20 & HB \\
\hline 32 & $10: 05: 32.63$ & $00: 04: 21.3$ & 20.39 & 0.37 & 0.02 & 0.01 & 0.18 & HB \\
\hline 33 & 10:05:35.91 & 00:05:10.3 & 20.39 & 0.39 & 0.01 & 0.01 & 1.32 & HB \\
\hline 34 & 10:05:33.32 & 00:04:38.0 & 20.40 & & & & 0.48 & HB \\
\hline 35 & 10:05: & 00:05:00.0 & 20.42 & & 0.0 & 0. & 1.30 & HB \\
\hline 36 & $10: 05: 39.49$ & 00:00:10.8 & 20.43 & 0.64 & 0.03 & 0.02 & 4.53 & AGB \\
\hline 37 & 10:05:34.47 & 00:03:57.1 & 20.43 & 0.45 & 0.01 & 0.01 & 0.72 & HB \\
\hline 38 & $10: 05: 27.77$ & $00: 04: 35.5$ & 20.43 & 0.43 & 0.01 & 0.01 & 1.09 & HB \\
\hline 39 & $10: 05: 30.44$ & 00:04:22.9 & 20.44 & 0.46 & 0.02 & 0.01 & 0.39 & $\mathrm{HB}$ \\
\hline 40 & $10: 05: 28.24$ & $00: 03: 34.3$ & 20.45 & 0.29 & 0.01 & 0.01 & 1.18 & HB \\
\hline 41 & $10: 05: 29.61$ & $00: 04: 21.3$ & 20.45 & 0.51 & 0.01 & 0.01 & 0.59 & HB \\
\hline 42 & $10: 05: 34.00$ & $00: 04: 27.4$ & 20.45 & 0.49 & 0.01 & 0.01 & 0.54 & $\mathrm{HB}$ \\
\hline 43 & $10: 05: 35.85$ & 00:04:15.8 & 20.46 & 0.44 & 0.01 & 0.01 & 0.98 & HB \\
\hline 44 & $10: 05: 29.65$ & $00: 04: 12.0$ & 20.47 & 0.35 & 0.04 & 0.01 & 0.58 & HB \\
\hline 45 & 10:05:27.39 & 00:05:09.7 & 20.47 & 0.38 & 0.02 & 0.01 & 1.43 & HB \\
\hline 46 & $10: 05: 31.53$ & $00: 04: 40.5$ & 20.47 & & 0. & 0. & 0.39 & HB \\
\hline 47 & $10: 05: 28.80$ & $00: 04: 10.9$ & 20.48 & 0 . & 0.01 & 0. & 0.80 & HB \\
\hline 48 & $10: 05: 29.12$ & $00: 03: 45.8$ & 20.49 & 0.49 & 0.02 & 0.0 & 0.89 & HB \\
\hline 49 & $10: 05$ & 00:0 & 20.5 & 0.4 & 0.01 & 0.01 & 1.28 & HB \\
\hline 50 & $10: 05: 3$ & $00: 04: 25.4$ & 20.51 & 0.75 & 0.02 & 0.01 & 0.13 & RGB \\
\hline 51 & $10: 05: 28.34$ & 00:04:48.7 & 20.54 & 0.76 & 0.01 & 0.01 & 1.04 & RGB \\
\hline 52 & $10: 05: 36.69$ & $00: 03: 15.7$ & 20.54 & 0.39 & 0.01 & 0.01 & 1.58 & $\mathrm{HB}$ \\
\hline 53 & 10:05:31.54 & $00: 04: 21.7$ & 20.54 & 0.41 & 0.02 & 0.01 & 0.12 & HB \\
\hline 54 & 10:05:38.09 & $00: 04: 53.3$ & 20.61 & 0.74 & 0.01 & 0.01 & 1.64 & RGB \\
\hline 55 & $10: 05: 30.53$ & $00: 04: 37.3$ & 20.61 & 0.47 & 0.02 & 0.01 & 0.48 & $\mathrm{HB}$ \\
\hline 56 & 10:05:33.21 & 00:05:06.2 & 20.63 & 0.75 & 0.02 & 0.01 & 0.86 & RGB \\
\hline 57 & $10: 05: 35.28$ & $00: 03: 43.1$ & 20.70 & 0.40 & 0.01 & 0.01 & 1.02 & HB \\
\hline 58 & $10: 05$ & 00:0 & 20.70 & & & 0 & 4.08 & HB \\
\hline 59 & $10:($ & 00 & 20 & & & & 0.40 & $\mathrm{HB}$ \\
\hline 60 & & & & & & & 0.14 & RGB \\
\hline 61 & $10: 05: 20.31$ & 00:07:37.3 & 20.76 & 0.42 & 0.02 & 0.01 & 4.42 & $\mathrm{HB}$ \\
\hline 62 & $10: 05: 30.12$ & $00: 04: 46.0$ & 20.86 & 0.72 & 0.02 & 0.01 & 0.65 & RGB \\
\hline 63 & 10:05:30.87 & $00: 04: 39.9$ & 20.92 & 0.69 & 0.02 & 0.02 & 0.45 & RGB \\
\hline 64 & $10: 05: 31.51$ & $00: 04: 54.5$ & 20.95 & 0.68 & 0.02 & 0.01 & 0.62 & RGB \\
\hline 65 & $10: 05: 31.88$ & $00: 06: 17.2$ & 20.96 & 0.66 & 0.02 & 0.02 & 1.99 & RGB \\
\hline 66 & $10: 05: 25.51$ & 00:04:15.7 & 21.01 & 0.71 & 0.02 & 0.01 & 1.61 & RGB \\
\hline 67 & 10:05:35.85 & 00:04:54.5 & 21.02 & 0.70 & 0.01 & 0.01 & 1.15 & RGB \\
\hline 68 & $10: 05: 32.48$ & 00:04:13.6 & 21.03 & 0.72 & 0.02 & 0.02 & 0.15 & RGB \\
\hline 69 & $10: 05$ & 00:0 & 21.05 & 0.6 & 0. & 0.03 & 4.14 & RGB \\
\hline 70 & & $00: 0$ & & 0 . & 0. & 0.02 & 0.31 & RGB \\
\hline 71 & $10: 05: 29.63$ & $00: 04: 23.8$ & 21.10 & 0.71 & 0.02 & 0.02 & 0.59 & RGB \\
\hline 72 & 10:05:33.12 & $00: 04: 36.1$ & 21.14 & 0.73 & 0.03 & 0.02 & 0.42 & RGB \\
\hline 73 & 10:05:30.90 & 00:04:48.5 & 21.17 & 0.66 & 0.04 & 0.03 & 0.57 & RGB \\
\hline 74 & $10: 05: 33.18$ & 00:03:59.9 & 21.22 & 0.63 & 0.02 & 0.02 & 0.43 & RGB \\
\hline 75 & 10:05:30.90 & 00:04:33.9 & 21.28 & 0.63 & 0.02 & 0.02 & 0.37 & RGB \\
\hline
\end{tabular}

Table A.2. continued.

\begin{tabular}{|c|c|c|c|c|c|c|c|c|}
\hline Id & $\begin{array}{c}\alpha(2000) \\
{[\mathrm{h}: \mathrm{m}: \mathrm{s}]}\end{array}$ & $\begin{array}{c}\delta(2000) \\
{\left[{ }^{0}:^{\prime}:^{\prime \prime}\right]}\end{array}$ & $\begin{array}{c}V \\
{[\mathrm{mag}]}\end{array}$ & $\begin{array}{l}B-V \\
{[\mathrm{mag}]}\end{array}$ & $\begin{array}{c}\sigma_{V} \\
{[\mathrm{mag}]}\end{array}$ & $\begin{array}{c}\sigma_{B} \\
{[\mathrm{mag}]}\end{array}$ & $\begin{array}{c}\text { radius } \\
{\left[{ }^{\prime}\right]}\end{array}$ & Type \\
\hline 76 & $10: 05: 31.21$ & $00: 05: 03.3$ & 21.32 & 0.70 & 0.02 & 0.02 & 0.78 & RGB \\
\hline 77 & $10: 05: 32.30$ & $00: 03: 55.3$ & 21.36 & 0.73 & 0.02 & 0.03 & 0.39 & RGB \\
\hline 78 & 10:05:37.93 & 00:04:07.8 & 21.42 & 0.72 & 0.02 & 0.01 & 1.50 & RGB \\
\hline 79 & $10: 05: 32.77$ & $00: 04: 15.2$ & 21.43 & 0.66 & 0.02 & 0.02 & 0.21 & RGB \\
\hline 80 & $10: 05: 29.94$ & $00: 04: 28.5$ & 21.48 & 0.68 & 0.02 & 0.01 & 0.53 & RGB \\
\hline 81 & $10: 05: 33.44$ & $00: 04: 25.2$ & 21.51 & 0.70 & 0.02 & 0.02 & 0.39 & RGB \\
\hline 82 & $10: 05: 25.75$ & $00: 04: 20.8$ & 21.52 & 0.66 & 0.02 & 0.01 & 1.55 & RGB \\
\hline 83 & $10: 05: 30.78$ & $00: 04: 29.1$ & 21.57 & 0.61 & 0.03 & 0.02 & 0.35 & RGB \\
\hline 84 & $10: 05: 30.89$ & 00:03:51.0 & 21.58 & 0.65 & 0.03 & 0.02 & 0.52 & RGB \\
\hline 85 & $10: 05: 38.12$ & $00: 05: 00.2$ & 21.63 & 0.67 & 0.02 & 0.01 & 1.70 & RGB \\
\hline 86 & $10: 05: 29.29$ & $00: 04: 24.8$ & 21.66 & 0.69 & 0.02 & 0.02 & 0.68 & RGB \\
\hline 87 & $10: 05: 29.48$ & $00: 03: 20.0$ & 21.70 & 0.68 & 0.02 & 0.02 & 1.15 & RGB \\
\hline 88 & 10:05:32.08 & $00: 04: 16.7$ & 21.71 & 0.65 & 0.03 & 0.02 & 0.04 & RGB \\
\hline 89 & $10: 05: 32.02$ & 00:04:51.4 & 21.73 & 0.66 & 0.02 & 0.02 & 0.56 & RGB \\
\hline 90 & $10: 05: 28.88$ & $00: 04: 12.1$ & 21.76 & 0.66 & 0.03 & 0.02 & 0.77 & RGB \\
\hline 91 & 10:05:28.96 & $00: 04: 20.4$ & 21.77 & 0.69 & 0.02 & 0.01 & 0.75 & RGB \\
\hline 92 & $10: 05: 31.12$ & $00: 04: 32.2$ & 21.78 & 0.62 & 0.03 & 0.03 & 0.31 & RGB \\
\hline 93 & $10: 05: 32.00$ & $00: 04: 22.0$ & 21.80 & 0.69 & 0.03 & 0.03 & 0.07 & RGB \\
\hline 94 & $10: 05: 30.37$ & 00:04:03.1 & 21.81 & 0.65 & 0.05 & 0.04 & 0.47 & RGB \\
\hline 95 & $10: 05: 33.34$ & $00: 04: 18.2$ & 21.83 & 0.65 & 0.04 & 0.02 & 0.35 & RGB \\
\hline 96 & $10: 05: 31.61$ & $00: 04: 12.2$ & 21.88 & 0.62 & 0.04 & 0.03 & 0.13 & RGB \\
\hline 97 & $10: 05: 32.43$ & 00:03:59.8 & 21.89 & 0.59 & 0.03 & 0.02 & 0.33 & RGB \\
\hline 98 & $10: 05: 29.36$ & $00: 08: 33.4$ & 21.92 & 0.66 & 0.04 & 0.03 & 4.31 & RGB \\
\hline 99 & $10: 05: 31.72$ & $00: 04: 19.8$ & 21.92 & 0.67 & 0.03 & 0.02 & 0.07 & RGB \\
\hline 100 & $10: 05: 35.42$ & 00:03:07.8 & 21.92 & 0.62 & 0.02 & 0.02 & 1.46 & RGB \\
\hline 101 & $10: 05: 33.85$ & $00: 06: 17.2$ & 21.93 & 0.64 & 0.03 & 0.03 & 2.04 & RGB \\
\hline 102 & $10: 05: 33.59$ & $00: 02: 53.0$ & 21.93 & 0.65 & 0.02 & 0.01 & 1.48 & RGB \\
\hline 103 & $10: 05: 30.03$ & $00: 04: 28.1$ & 21.94 & 0.70 & 0.02 & 0.02 & 0.51 & RGB \\
\hline 104 & $10: 05: 32.34$ & $00: 04: 21.4$ & 21.98 & 0.63 & 0.03 & 0.03 & 0.11 & RGB \\
\hline 105 & $10: 05: 33.53$ & 00:04:20.9 & 21.99 & 0.68 & 0.03 & 0.02 & 0.40 & RGB \\
\hline 106 & $10: 05: 29.06$ & $00: 04: 29.4$ & 22.02 & 0.60 & 0.03 & 0.02 & 0.75 & RGB \\
\hline 107 & $10: 05: 37.11$ & 00:04:58.6 & 22.02 & 0.65 & 0.02 & 0.02 & 1.46 & RGB \\
\hline 108 & $10: 05: 36.87$ & $00: 03: 42.5$ & 22.04 & 0.64 & 0.03 & 0.03 & 1.36 & RGB \\
\hline 109 & 10:05:33.07 & 00:04:09.4 & 22.04 & 0.69 & 0.03 & 0.02 & 0.32 & RGB \\
\hline 110 & $10: 05: 23.81$ & $00: 04: 22.7$ & 22.07 & 0.64 & 0.02 & 0.02 & 2.04 & RGB \\
\hline 111 & $10: 05: 40.33$ & $00: 09: 14.9$ & 22.09 & 0.60 & 0.04 & 0.03 & 5.37 & RGB \\
\hline 112 & $10: 05: 27.96$ & $00: 04: 26.0$ & 22.10 & 0.71 & 0.03 & 0.02 & 1.01 & RGB \\
\hline 113 & $10: 05: 21.85$ & 00:07:41.0 & 22.10 & 0.58 & 0.04 & 0.03 & 4.22 & RGB \\
\hline 114 & $10: 05: 41.53$ & $00: 05: 45.4$ & 22.14 & 0.67 & 0.05 & 0.03 & 2.80 & RGB \\
\hline 115 & 10:05:33.00 & $00: 06: 34.6$ & 22.16 & 0.59 & 0.04 & 0.03 & 2.29 & RGB \\
\hline 116 & $10: 05: 31.59$ & $00: 03: 28.9$ & 22.17 & 0.59 & 0.03 & 0.02 & 0.82 & RGB \\
\hline 117 & $10: 05: 29.98$ & $00: 04: 27.1$ & 22.18 & 0.64 & 0.04 & 0.04 & 0.52 & RGB \\
\hline 118 & $10: 05: 28.18$ & $00: 04: 35.3$ & 22.18 & 0.57 & 0.03 & 0.02 & 0.98 & RGB \\
\hline 119 & $10: 05: 28.65$ & $00: 05: 48.8$ & 22.18 & 0.65 & 0.03 & 0.02 & 1.72 & RGB \\
\hline 120 & $10: 05: 34.50$ & $00: 04: 42.8$ & 22.20 & 0.70 & 0.03 & 0.02 & 0.76 & RGB \\
\hline 121 & $10: 05: 30.95$ & $00: 04: 12.4$ & 22.21 & 0.59 & 0.04 & 0.03 & 0.27 & RGB \\
\hline 122 & $10: 05: 33.34$ & $00: 05: 35.4$ & 22.23 & 0.70 & 0.04 & 0.04 & 1.34 & RGB \\
\hline 123 & $10: 05: 32.15$ & 00:04:51.1 & 22.23 & 0.63 & 0.04 & 0.02 & 0.55 & RGB \\
\hline 124 & $10: 05: 30.30$ & 00:04:07.6 & 22.23 & 0.63 & 0.04 & 0.03 & 0.45 & RGB \\
\hline 125 & 10:05:33.96 & $00: 03: 30.5$ & 22.24 & 0.63 & 0.04 & 0.03 & 0.94 & RGB \\
\hline 126 & $10: 05: 27.88$ & $00: 08: 29.6$ & 22.26 & 0.60 & 0.05 & 0.04 & 4.31 & RGB \\
\hline 127 & 10:05:31.40 & $00: 03: 25.4$ & 22.26 & 0.64 & 0.05 & 0.03 & 0.89 & RGB \\
\hline 128 & 10:05:31.84 & $00: 04: 42.6$ & 22.27 & 0.70 & 0.04 & 0.03 & 0.41 & RGB \\
\hline 129 & $10: 05: 30.64$ & $00: 03: 16.8$ & 22.30 & 0.63 & 0.04 & 0.03 & 1.07 & RGB \\
\hline 130 & 10:05:31.42 & 00:03:36.1 & 22.30 & 0.65 & 0.04 & 0.03 & 0.71 & RGB \\
\hline 131 & $10: 05: 32.78$ & 00:04:09.7 & 22.30 & 0.64 & 0.06 & 0.04 & 0.25 & RGB \\
\hline 132 & $10: 05: 29.59$ & $00: 04: 26.3$ & 22.34 & 0.64 & 0.03 & 0.03 & 0.61 & RGB \\
\hline 133 & $10: 05: 31.92$ & 00:05:08.9 & 22.35 & 0.69 & 0.05 & 0.04 & 0.85 & RGB \\
\hline 134 & $10: 05: 34.04$ & $00: 03: 44.3$ & 22.35 & 0.56 & 0.04 & 0.04 & 0.77 & RGB \\
\hline 135 & $10: 05: 28.01$ & 00:04:06.1 & 22.36 & 0.66 & 0.04 & 0.03 & 1.00 & RGB \\
\hline 136 & $10: 05: 32.55$ & $00: 03: 34.7$ & 22.39 & 0.67 & 0.04 & 0.03 & 0.74 & RGB \\
\hline 137 & $10: 05: 32.12$ & 00:04:57.7 & 22.40 & 0.71 & 0.06 & 0.05 & 0.66 & RGB \\
\hline 138 & $10: 05: 29.26$ & $00: 03: 44.5$ & 22.41 & 0.62 & 0.04 & 0.03 & 0.88 & RGB \\
\hline 139 & $10: 05: 30.79$ & $00: 04: 24.1$ & 22.44 & 0.62 & 0.05 & 0.03 & 0.31 & RGB \\
\hline 140 & 10:05:36.89 & $00: 06: 35.1$ & 22.44 & 0.56 & 0.05 & 0.03 & 2.60 & RGB \\
\hline 141 & 10:05:37.92 & 00:02:41.4 & 22.45 & 0.57 & 0.07 & 0.07 & 2.20 & RGB \\
\hline 142 & $10: 05: 30.71$ & $00: 03: 46.7$ & 22.48 & 0.66 & 0.04 & 0.04 & 0.61 & RGB \\
\hline 143 & $10: 05: 32.34$ & 00:04:05.6 & 22.48 & 0.62 & 0.04 & 0.04 & 0.23 & RGB \\
\hline 144 & $10: 05: 33.16$ & 00:04:33.6 & 22.48 & 0.71 & 0.05 & 0.04 & 0.40 & RGB \\
\hline 145 & $10: 05: 33.91$ & $00: 04: 48.9$ & 22.48 & 0.59 & 0.03 & 0.02 & 0.71 & RGB \\
\hline 146 & $10: 05: 30.26$ & $00: 03: 45.6$ & 22.52 & 0.69 & 0.05 & 0.04 & 0.69 & RGB \\
\hline 147 & $10: 05: 37.41$ & 00:06:11.1 & 22.52 & 0.56 & 0.05 & 0.03 & 2.33 & RGB \\
\hline 148 & $10: 05: 28.19$ & $00: 03: 26.2$ & 22.52 & 0.66 & 0.04 & 0.04 & 1.28 & RGB \\
\hline 149 & $10: 05: 24.98$ & $00: 04: 14.3$ & 22.52 & 0.69 & 0.04 & 0.04 & 1.74 & RGB \\
\hline 150 & 10:05:32.97 & $00: 04: 37.8$ & 22.53 & 0.55 & 0.05 & 0.03 & 0.42 & RGB \\
\hline 151 & 10:05:32.30 & $00: 03: 22.6$ & 22.60 & 0.67 & 0.05 & 0.03 & 0.93 & RGB \\
\hline 152 & $10: 05: 27.74$ & $00: 03: 19.7$ & 22.62 & 0.59 & 0.04 & 0.04 & 1.43 & RGB \\
\hline 153 & 10:05:29.79 & $00: 03: 46.0$ & 22.62 & 0.67 & 0.05 & 0.04 & 0.76 & RGB \\
\hline
\end{tabular}


M. Hilker: Probable member stars of AM 1, Pal 3 and Pal 14, Online Material p 4

Table A.3. List of probable member stars of Pal 14, ordered by in- Table A.3. continued. creasing $V$ magnitude.

\begin{tabular}{|c|c|c|c|c|c|c|c|c|}
\hline Id & $\begin{array}{c}\alpha(2000) \\
{[\mathrm{h}: \mathrm{m}: \mathrm{s}]}\end{array}$ & $\begin{array}{c}(2000) \\
{\left[:^{\circ}:^{\prime \prime}\right]}\end{array}$ & $\begin{array}{c}V \\
{[\mathrm{mag}]}\end{array}$ & $\begin{array}{l}B-V \\
{[\mathrm{mag}]}\end{array}$ & $\begin{array}{c}\sigma_{V} \\
{[\mathrm{mag}]}\end{array}$ & $\begin{array}{c}\sigma_{B} \\
{[\mathrm{mag}]}\end{array}$ & $\begin{array}{c}\text { radius } \\
{\left[{ }^{\prime}\right]}\end{array}$ & Type \\
\hline 1 & $16: 11: 05.81$ & $14: 57: 45.1$ & 17.37 & 1.39 & 0.02 & 0.01 & 1.28 & RGB \\
\hline 2 & $16: 10: 58.73$ & $14: 56: 48.7$ & 17.77 & 1.29 & 0.02 & 0.01 & 0.80 & RGB \\
\hline 3 & $16: 10: 54.90$ & $14: 58: 36.7$ & 18.23 & 1.07 & 0.01 & 0.01 & 1.80 & AGB? \\
\hline 4 & $16: 11: 04.98$ & $14: 53: 32.3$ & 18.48 & 1.10 & 0.02 & 0.01 & 4.07 & RGB \\
\hline 5 & $16: 10: 59.24$ & $14: 57: 22.5$ & 18.52 & 0.99 & 0.01 & 0.01 & 0.35 & AGB? \\
\hline 6 & $16: 10: 53.36$ & $14: 56: 45.4$ & 18.56 & 1.04 & 0.01 & 0.01 & 1.90 & RGB \\
\hline 7 & $16: 10: 54.04$ & $14: 57: 05.6$ & 18.70 & 1.03 & 0.01 & 0.01 & 1.64 & RGB \\
\hline 8 & $16: 10: 56.90$ & $14: 57: 56.5$ & 18.84 & 0.99 & 0.01 & 0.01 & 1.02 & RGB \\
\hline 9 & $16: 11: 01.40$ & $14: 57: 60.0$ & 19.05 & 0.99 & 0.01 & 0.01 & 0.56 & RGB \\
\hline 10 & $16: 11: 05.89$ & $14: 58: 43.2$ & 19.19 & 0.93 & 0.02 & 0.01 & 1.78 & RGB \\
\hline 11 & $16: 11: 06.00$ & $14: 55: 39.1$ & 19.37 & 0.95 & 0.01 & 0.01 & 2.23 & RGB \\
\hline 12 & $16: 10: 56.21$ & $14: 56: 32.7$ & 19.41 & 0.90 & 0.01 & 0.01 & 1.42 & RGB \\
\hline 13 & $16: 10: 56.98$ & $14: 56: 25.8$ & 19.44 & 0.92 & 0.01 & 0.01 & 1.37 & RGB \\
\hline 14 & $16: 10: 59.24$ & $14: 57: 19.7$ & 19.50 & 0.90 & 0.02 & 0.01 & 0.37 & RGB \\
\hline 15 & $16: 10: 55.84$ & $14: 57: 43.4$ & 19.60 & 0.69 & 0.01 & 0.01 & 1.19 & AGB \\
\hline 16 & $16: 10: 59.62$ & $15: 01: 32.9$ & 19.68 & 0.81 & 0.02 & 0.01 & 4.09 & RGB \\
\hline 17 & $16: 11: 00.58$ & 14:56:59.1 & 19.76 & 0.86 & 0.01 & 0.01 & 0.48 & RGB \\
\hline 18 & $16: 11: 03.91$ & $14: 56: 34.3$ & 19.77 & 0.59 & 0.01 & 0.01 & 1.19 & AGB \\
\hline 19 & $16: 11: 01.01$ & $14: 55: 23.1$ & 19.78 & 0.69 & 0.01 & 0.01 & 2.09 & AGB \\
\hline 20 & $16: 11: 07.42$ & $14: 57: 43.2$ & 19.86 & 0.86 & 0.01 & 0.01 & 1.65 & RGB \\
\hline 21 & $16: 10: 52.81$ & $14: 55: 38.9$ & 19.87 & 0.84 & 0.01 & 0.01 & 2.62 & RGB \\
\hline 22 & $16: 10: 56.17$ & $14: 55: 48.1$ & 19.88 & 0.46 & 0.01 & 0.01 & 1.99 & HB \\
\hline 23 & $16: 11: 02.52$ & $14: 56: 49.7$ & 19.88 & 0.62 & 0.01 & 0.01 & 0.78 & HB \\
\hline 24 & $16: 11: 10.09$ & $14: 55: 54.9$ & 19.89 & 0.88 & 0.02 & 0.01 & 2.76 & RGB \\
\hline 25 & $16: 11: 04.45$ & $14: 56: 14.4$ & 19.90 & 0.65 & 0.01 & 0.01 & 1.53 & HB \\
\hline 26 & $16: 10: 59.32$ & $14: 57: 25.9$ & 19.92 & 0.84 & 0.02 & 0.01 & 0.32 & RGB \\
\hline 27 & $16: 11: 16.22$ & 15:00:07.1 & 19.92 & 0.47 & 0.02 & 0.01 & 4.60 & $\mathrm{HB}$ \\
\hline 28 & $16: 10: 59.89$ & $14: 53: 27.8$ & 19.92 & 0.92 & 0.03 & 0.02 & 4.01 & RGB \\
\hline 29 & $16: 11: 12.60$ & $14: 56: 55.2$ & 19.93 & 0.46 & 0.02 & 0.01 & 2.94 & HB \\
\hline 30 & $16: 11: 13.56$ & $14: 57: 58.2$ & 19.95 & 0.85 & 0.02 & 0.01 & 3.16 & RGB \\
\hline 31 & $16: 11: 01.64$ & $14: 59: 47.8$ & 19.95 & 0.80 & 0.02 & 0.02 & 2.34 & RGB \\
\hline 32 & $16: 10: 56.39$ & $14: 57: 25.1$ & 19.96 & 0.84 & 0.01 & 0.02 & 1.03 & RGB \\
\hline 33 & $16: 11: 09.37$ & $14: 57: 37.5$ & 19.97 & 0.61 & 0.02 & 0.01 & 2.11 & HB \\
\hline 34 & $16: 10: 58.62$ & $14: 57: 40.3$ & 19.97 & 0.83 & 0.01 & 0.01 & 0.53 & RGB \\
\hline 35 & $16: 11: 09.57$ & $15: 01: 07.7$ & 19.98 & 0.58 & 0.02 & 0.02 & 4.25 & HB \\
\hline 36 & $16: 10: 59.15$ & $14: 57: 38.0$ & 19.98 & 0.76 & 0.01 & 0.01 & 0.40 & HB? \\
\hline 37 & $16: 10: 56.20$ & $14: 57: 34.4$ & 19.99 & 0.61 & 0.01 & 0.01 & 1.08 & HB \\
\hline 38 & $16: 11: 01.76$ & $14: 58: 06.8$ & 19.99 & 0.61 & 0.01 & 0.01 & 0.70 & HB \\
\hline 39 & $16: 10: 59.72$ & $14: 57: 45.6$ & 20.00 & 0.58 & 0.01 & 0.01 & 0.37 & HB \\
\hline 40 & $16: 10: 59.15$ & $14: 55: 01.8$ & 20.03 & 0.67 & 0.02 & 0.02 & 2.46 & $\mathrm{HB}$ \\
\hline 41 & $16: 11: 00.81$ & $14: 57: 53.4$ & 20.03 & 0.58 & 0.02 & 0.01 & 0.42 & HB \\
\hline 42 & $16: 10: 53.24$ & $14: 57: 50.2$ & 20.03 & 0.55 & 0.01 & 0.01 & 1.83 & HB \\
\hline 43 & $16: 10: 53.08$ & $14: 56: 22.9$ & 20.03 & 0.55 & 0.02 & 0.01 & 2.13 & $\mathrm{HB}$ \\
\hline 44 & $16: 10: 55.09$ & $14: 57: 44.3$ & 20.04 & 0.60 & 0.01 & 0.01 & 1.37 & $\mathrm{HB}$ \\
\hline 45 & 16:11:01.49 & $14: 57: 46.0$ & 20.04 & 0.58 & 0.02 & 0.01 & 0.36 & HB \\
\hline 46 & $16: 11: 08.53$ & $14: 53: 09.0$ & 20.04 & 0.50 & 0.02 & 0.01 & 4.72 & $\mathrm{HB}$ \\
\hline 47 & $16: 10: 53.97$ & $14: 56: 35.4$ & 20.06 & 0.55 & 0.01 & 0.02 & 1.84 & HB \\
\hline 48 & $16: 11: 03.24$ & $14: 57: 44.9$ & 20.06 & 0.62 & 0.01 & 0.01 & 0.69 & $\mathrm{HB}$ \\
\hline 49 & $16: 11: 16.01$ & $14: 54: 36.2$ & 20.07 & 0.64 & 0.03 & 0.02 & 4.69 & HB \\
\hline 50 & $16: 11: 02.83$ & 14:57:09.0 & 20.07 & 0.56 & 0.01 & 0.01 & 0.62 & HB \\
\hline 51 & $16: 10: 59.37$ & $14: 57: 43.5$ & 20.07 & 0.57 & 0.01 & 0.01 & 0.40 & HB \\
\hline 52 & $16: 10: 53.27$ & $14: 56: 27.2$ & 20.07 & 0.54 & 0.01 & 0.01 & 2.05 & HB \\
\hline 53 & $16: 11: 00.38$ & $14: 57: 40.7$ & 20.08 & 0.55 & 0.02 & 0.01 & 0.22 & HB \\
\hline 54 & 16:11:09.51 & $14: 59: 43.9$ & 20.08 & 0.57 & 0.02 & 0.01 & 3.12 & $\mathrm{HB}$ \\
\hline 55 & $16: 10: 59.25$ & $14: 57: 14.0$ & 20.08 & 0.58 & 0.01 & 0.01 & 0.41 & HB \\
\hline 56 & $16: 10: 59.65$ & $14: 56: 43.4$ & 20.11 & 0.59 & 0.01 & 0.02 & 0.78 & HB \\
\hline 57 & 16:11:00.94 & $14: 58: 50.3$ & 20.11 & 0.41 & 0.01 & 0.01 & 1.37 & HB \\
\hline 58 & $16: 11: 01.91$ & $14: 57: 53.2$ & 20.11 & 0.84 & 0.01 & 0.02 & 0.52 & RGB \\
\hline 59 & $16: 10: 56.97$ & $14: 57: 28.0$ & 20.11 & 0.56 & 0.01 & 0.01 & 0.89 & HB \\
\hline 60 & $16: 10: 57.39$ & $14: 56: 01.3$ & 20.13 & 0.86 & 0.02 & 0.02 & 1.65 & RGB \\
\hline 61 & $16: 11: 01.71$ & $14: 57: 36.9$ & 20.14 & 0.57 & 0.01 & 0.01 & 0.30 & HB \\
\hline 62 & $16: 10: 59.47$ & 14:56:03.6 & 20.19 & 0.67 & 0.01 & 0.01 & 1.44 & $\mathrm{HB}$ \\
\hline 63 & $16: 11: 13.75$ & $14: 54: 18.3$ & 20.24 & 0.88 & 0.04 & 0.02 & 4.48 & RGB \\
\hline 64 & $16: 10: 59.47$ & 14:58:02.1 & 20.28 & 0.84 & 0.02 & 0.02 & 0.63 & RGB \\
\hline 65 & $16: 10: 59.65$ & $14: 55: 42.4$ & 20.30 & 0.79 & 0.01 & 0.02 & 1.78 & RGB \\
\hline 66 & $16: 10: 58.97$ & $14: 55: 28.4$ & 20.33 & 0.82 & 0.02 & 0.01 & 2.04 & RGB \\
\hline 67 & $16: 10: 54.49$ & $14: 57: 19.5$ & 20.34 & 0.82 & 0.01 & 0.01 & 1.49 & RGB \\
\hline 68 & $16: 10: 59.66$ & $14: 58: 18.5$ & 20.38 & 0.83 & 0.02 & 0.02 & 0.87 & RGB \\
\hline 69 & $16: 11: 01.04$ & $14: 58: 32.6$ & 20.39 & 0.83 & 0.02 & 0.02 & 1.08 & RGB \\
\hline 70 & $16: 11: 15.80$ & $14: 57: 55.6$ & 20.41 & 0.91 & 0.02 & 0.02 & 3.69 & RGB \\
\hline
\end{tabular}

\begin{tabular}{|c|c|c|c|c|c|c|c|c|}
\hline Id & $\begin{array}{c}\alpha(2000) \\
{[\mathrm{h}: \mathrm{m}: \mathrm{s}]}\end{array}$ & $\begin{array}{c}\delta(2000) \\
{\left[{ }^{\circ}:^{\prime}:^{\prime \prime}\right]}\end{array}$ & $\begin{array}{c}V \\
{[\mathrm{mag}]}\end{array}$ & $\begin{array}{l}B-V \\
{[\mathrm{mag}]}\end{array}$ & $\begin{array}{c}\sigma_{V} \\
{[\mathrm{mag}]}\end{array}$ & $\begin{array}{c}\sigma_{B} \\
{[\mathrm{mag}]}\end{array}$ & $\begin{array}{c}\text { radius } \\
\text { ['] }\end{array}$ & Type \\
\hline 71 & $16: 10: 57.31$ & $14: 59: 21.7$ & 20.42 & 0.44 & 0.02 & 0.01 & 2.06 & HB? \\
\hline 72 & $16: 11: 08.15$ & $14: 57: 40.5$ & 20.48 & 0.77 & 0.02 & 0.02 & 1.82 & RGB \\
\hline 73 & $16: 11: 07.45$ & $14: 58: 30.7$ & 20.51 & 0.79 & 0.02 & 0.02 & 1.95 & RGB \\
\hline 74 & $16: 11: 03.28$ & $14: 58: 04.1$ & 20.54 & 0.81 & 0.02 & 0.02 & 0.87 & RGB \\
\hline 75 & $16: 10: 56.94$ & $14: 57: 10.5$ & 20.58 & 0.78 & 0.02 & 0.02 & 0.94 & RGB \\
\hline 76 & $16: 10: 57.67$ & $14: 57: 19.1$ & 20.60 & 0.74 & 0.01 & 0.02 & 0.73 & RGB \\
\hline 77 & $16: 10: 59.59$ & $14: 57: 20.1$ & 20.67 & 0.74 & 0.01 & 0.02 & 0.29 & RGB \\
\hline 78 & $16: 11: 11.43$ & $14: 58: 09.6$ & 20.67 & 0.81 & 0.02 & 0.02 & 2.69 & RGB \\
\hline 79 & $16: 11: 00.93$ & $14: 57: 31.7$ & 20.83 & 0.74 & 0.02 & 0.03 & 0.09 & RGB \\
\hline 80 & $16: 10: 58.97$ & $14: 53: 23.0$ & 20.85 & 0.81 & 0.03 & 0.02 & 4.11 & RGB \\
\hline 81 & $16: 11: 07.10$ & $14: 58: 17.8$ & 20.85 & 0.78 & 0.02 & 0.02 & 1.76 & RGB \\
\hline 82 & $16: 11: 01.63$ & $14: 55: 27.6$ & 20.90 & 0.77 & 0.02 & 0.03 & 2.02 & RGB \\
\hline 83 & $16: 11: 00.90$ & $14: 58: 06.8$ & 20.97 & 0.70 & 0.02 & 0.03 & 0.65 & RGB \\
\hline 84 & $16: 10: 59.36$ & $14: 56: 58.3$ & 20.98 & 0.76 & 0.02 & 0.03 & 0.59 & RGB \\
\hline 85 & $16: 11: 17.29$ & $14: 56: 19.9$ & 21.00 & 0.72 & 0.02 & 0.02 & 4.18 & RGB \\
\hline 86 & $16: 10: 54.40$ & $14: 58: 31.1$ & 21.02 & 0.74 & 0.02 & 0.02 & 1.84 & RGB \\
\hline 87 & $16: 10: 54.08$ & $14: 58: 33.6$ & 21.05 & 0.77 & 0.02 & 0.03 & 1.93 & RGB \\
\hline 88 & $16: 11: 02.26$ & $14: 58: 25.2$ & 21.10 & 0.82 & 0.02 & 0.04 & 1.03 & RGB \\
\hline 89 & $16: 11: 06.41$ & $14: 53: 22.1$ & 21.12 & 0.85 & 0.02 & 0.04 & 4.33 & RGB \\
\hline 90 & $16: 11: 09.75$ & $14: 57: 55.8$ & 21.18 & 0.75 & 0.02 & 0.04 & 2.25 & RGB \\
\hline 91 & $16: 11: 05.62$ & $14: 58: 45.8$ & 21.18 & 0.69 & 0.02 & 0.03 & 1.77 & RGB \\
\hline 92 & $16: 11: 07.25$ & $14: 57: 11.9$ & 21.21 & 0.79 & 0.02 & 0.04 & 1.62 & RGB \\
\hline 93 & $16: 11: 02.05$ & $14: 58: 21.3$ & 21.21 & 0.70 & 0.02 & 0.04 & 0.95 & RGB \\
\hline 94 & $16: 10: 57.26$ & $14: 57: 35.4$ & 21.23 & 0.71 & 0.02 & 0.03 & 0.83 & RGB \\
\hline 95 & $16: 11: 00.23$ & $14: 58: 02.6$ & 21.23 & 0.72 & 0.02 & 0.03 & 0.58 & RGB \\
\hline 96 & $16: 10: 59.37$ & $14: 57: 35.3$ & 21.24 & 0.72 & 0.02 & 0.03 & 0.33 & RGB \\
\hline 97 & $16: 11: 03.92$ & $14: 56: 21.7$ & 21.29 & 0.70 & 0.03 & 0.03 & 1.36 & RGB \\
\hline 98 & $16: 10: 59.12$ & $14: 56: 52.0$ & 21.30 & 0.74 & 0.02 & 0.04 & 0.70 & RGB \\
\hline 99 & $16: 11: 05.63$ & $14: 59: 20.3$ & 21.30 & 0.76 & 0.03 & 0.05 & 2.22 & RGB \\
\hline 100 & $16: 11: 00.25$ & $14: 58: 12.4$ & 21.35 & 0.78 & 0.03 & 0.04 & 0.75 & RGB \\
\hline 101 & $16: 11: 08.20$ & $14: 58: 53.5$ & 21.40 & 0.69 & 0.02 & 0.03 & 2.31 & RGB \\
\hline 102 & $16: 10: 59.70$ & $14: 57: 29.8$ & 21.41 & 0.77 & 0.02 & 0.04 & 0.23 & RGB \\
\hline 103 & $16: 11: 01.68$ & $14: 57: 09.4$ & 21.42 & 0.73 & 0.02 & 0.04 & 0.40 & RGB \\
\hline 104 & $16: 10: 58.82$ & $14: 57: 17.6$ & 21.43 & 0.76 & 0.02 & 0.04 & 0.47 & RGB \\
\hline 105 & $16: 11: 00.37$ & $14: 57: 45.6$ & 21.47 & 0.73 & 0.03 & 0.04 & 0.30 & RGB \\
\hline 106 & 16:11:01.58 & $14: 58: 49.8$ & 21.48 & 0.74 & 0.02 & 0.04 & 1.38 & RGB \\
\hline 107 & $16: 10: 57.76$ & $14: 55: 27.3$ & 21.54 & 0.81 & 0.03 & 0.05 & 2.13 & RGB \\
\hline 108 & $16: 11: 00.59$ & $14: 57: 03.1$ & 21.56 & 0.67 & 0.03 & 0.05 & 0.42 & RGB \\
\hline 109 & $16: 11: 01.72$ & $14: 57: 56.4$ & 21.59 & 0.75 & 0.02 & 0.04 & 0.54 & RGB \\
\hline 110 & $16: 11: 03.46$ & $14: 56: 35.5$ & 21.65 & 0.75 & 0.02 & 0.05 & 1.11 & RGB \\
\hline 111 & $16: 11: 02.28$ & $14: 58: 13.4$ & 21.66 & 0.63 & 0.03 & 0.04 & 0.85 & RGB \\
\hline 112 & $16: 11: 00.03$ & $14: 57: 27.7$ & 21.72 & 0.78 & 0.03 & 0.05 & 0.15 & RGB \\
\hline 113 & $16: 10: 55.84$ & $14: 56: 54.1$ & 21.72 & 0.73 & 0.03 & 0.06 & 1.29 & RGB \\
\hline 114 & $16: 11: 15.66$ & $14: 59: 26.6$ & 21.74 & 0.74 & 0.03 & 0.06 & 4.13 & RGB \\
\hline 115 & $16: 11: 02.13$ & $14: 57: 17.1$ & 21.75 & 0.79 & 0.05 & 0.05 & 0.40 & RGB \\
\hline 116 & $16: 11: 01.79$ & $14: 55: 48.3$ & 21.76 & 0.68 & 0.03 & 0.05 & 1.68 & RGB \\
\hline 117 & $16: 11: 00.66$ & $14: 57: 03.8$ & 21.78 & 0.68 & 0.03 & 0.04 & 0.40 & RGB \\
\hline 118 & $16: 10: 58.93$ & $14: 57: 53.1$ & 21.80 & 0.70 & 0.02 & 0.05 & 0.59 & RGB \\
\hline 119 & $16: 11: 01.50$ & $14: 58: 28.3$ & 21.82 & 0.71 & 0.03 & 0.05 & 1.02 & RGB \\
\hline 120 & $16: 10: 57.79$ & $14: 56: 23.9$ & 21.83 & 0.69 & 0.03 & 0.05 & 1.27 & RGB \\
\hline 121 & $16: 11: 04.03$ & 15:02:01.1 & 21.84 & 0.78 & 0.03 & 0.06 & 4.62 & RGB \\
\hline 122 & $16: 10: 58.53$ & $14: 57: 02.2$ & 21.85 & 0.65 & 0.03 & 0.05 & 0.67 & RGB \\
\hline 123 & $16: 11: 01.37$ & $14: 57: 40.2$ & 21.87 & 0.78 & 0.03 & 0.05 & 0.27 & RGB \\
\hline 124 & $16: 10: 55.65$ & $14: 57: 12.9$ & 21.92 & 0.64 & 0.03 & 0.06 & 1.23 & RGB \\
\hline 125 & $16: 11: 01.20$ & $14: 58: 37.3$ & 21.92 & 0.66 & 0.02 & 0.04 & 1.16 & RGB \\
\hline 126 & $16: 11: 00.14$ & $14: 58: 22.3$ & 21.93 & 0.67 & 0.04 & 0.06 & 0.91 & RGB \\
\hline 127 & $16: 11: 05.39$ & 14:56:59.9 & 21.94 & 0.69 & 0.04 & 0.07 & 1.24 & RGB \\
\hline 128 & $16: 10: 55.79$ & $14: 58: 02.7$ & 22.00 & 0.72 & 0.04 & 0.06 & 1.31 & RGB \\
\hline 129 & $16: 10: 59.39$ & 15:01:17.9 & 22.01 & 0.62 & 0.04 & 0.05 & 3.84 & RGB \\
\hline 130 & $16: 11: 00.63$ & $14: 58: 27.8$ & 22.01 & 0.78 & 0.03 & 0.07 & 0.99 & RGB \\
\hline 131 & $16: 10: 59.19$ & $14: 56: 02.5$ & 22.02 & 0.71 & 0.03 & 0.08 & 1.47 & RGB \\
\hline 132 & $16: 11: 10.01$ & $14: 58: 51.7$ & 22.06 & 0.75 & 0.02 & 0.04 & 2.66 & RGB \\
\hline 133 & $16: 11: 02.13$ & $14: 57: 03.0$ & 22.08 & 0.72 & 0.03 & 0.07 & 0.55 & RGB \\
\hline 134 & $16: 10: 58.95$ & $14: 58: 04.7$ & 22.10 & 0.61 & 0.04 & 0.07 & 0.74 & RGB \\
\hline 135 & $16: 10: 55.42$ & $14: 57: 20.6$ & 22.11 & 0.69 & 0.04 & 0.07 & 1.27 & RGB \\
\hline 136 & $16: 10: 57.33$ & $14: 57: 23.4$ & 22.14 & 0.61 & 0.05 & 0.07 & 0.81 & RGB \\
\hline 137 & $16: 11: 03.62$ & $14: 57: 22.5$ & 22.15 & 0.76 & 0.04 & 0.07 & 0.72 & RGB \\
\hline 138 & 16:11:00.66 & 14:56:51.7 & 22.18 & 0.68 & 0.04 & 0.08 & 0.61 & RGB \\
\hline 139 & $16: 11: 00.83$ & $14: 58: 22.2$ & 22.24 & 0.69 & 0.04 & 0.08 & 0.90 & RGB \\
\hline 140 & $16: 11: 01.28$ & $14: 56: 51.2$ & 22.27 & 0.71 & 0.04 & 0.11 & 0.63 & RGB \\
\hline 141 & $16: 10: 54.85$ & $15: 00: 45.2$ & 22.29 & 0.70 & 0.05 & 0.09 & 3.57 & RGB \\
\hline 142 & $16: 11: 03.71$ & $14: 57: 16.6$ & 22.30 & 0.78 & 0.04 & 0.09 & 0.76 & RGB \\
\hline 143 & 16:11:04.49 & $14: 57: 38.2$ & 22.31 & 0.70 & 0.05 & 0.09 & 0.94 & RGB \\
\hline
\end{tabular}

